This is a postprint version of the following published document:

Merino, M. Ahedo, E. Magnetic nozzles for space plasma thrusters, In:

Encyclopedia of plasma technology, vol. 2. CRC, 2016, Pp. 1329-1351

URL:

https://www.taylorfrancis.com/books/e/9781351204958/chapters/10.10 81/E-EPLT-120053936

(C) 2017 Taylor \& Francis Group, LLC. 


\title{
Magnetic Nozzles for Space Plasma Thrusters
}

\author{
Mario Merino, Eduardo Ahedo
}

November 11, 2015

\begin{abstract}
The magnetic nozzle is an appealing plasma acceleration technology that enables the electrodeless, contactless and adaptable control of a plasma jet. Using a two-dimensional, two-fluid plasma model, the core physical principles that enable the operation of a propulsive magnetic nozzle are reviewed, including the key mechanisms involved in thrust generation, plasma acceleration, and plasma detachment. Simulations hint at the parametric regimes that maximize the propulsive performance of the device. Extensions of the model are used to explore additional physical effects and their influence on the plasma expansion. While there are still open questions to address, magnetic nozzles appear to be a promising candidate for high-efficiency, high-durability plasma propulsion in future space missions.
\end{abstract}

\section{Contents}

\section{Introduction $\quad 2$}

2 Fundamental physics of magnetic nozzles 5

2.1 Model of the plasma flow in a magnetic nozzle . . . . . . . . 6

2.2 Electron fluid and supersonic expansion of ions . . . . . . . . 10

2.3 Energy conversion and thrust generation . . . . . . . . . . 13

2.4 Plasma detachment from the magnetic field . . . . . . . . . 17

$\begin{array}{lll}3 & \text { Propulsive performance } & 19\end{array}$

3.1 Thrust gain and plume efficiency functions . . . . . . . . . 20

3.2 Plume divergence . . . . . . . . . . . . . . . . . 22

4 Advanced physics of magnetic nozzles 23

4.1 On the thermodynamics of electrons . . . . . . . . . . 23

4.2 Influence of the type of internal energy in the plasma . . . . . . 27

4.3 Plasma-induced magnetic field . . . . . . . . . . . . . . 29

4.4 Collisional processes in the expansion . . . . . . . . . . . 31

4.5 Influence of electron inertia . . . . . . . . . . . . . . . 34 
5.1 Open areas of research . . . . . . . . . . . . . . . 36

\section{Introduction}

A magnetic nozzle $(\mathrm{MN})$ consists in a convergent-divergent, applied magnetic field capable of guiding and accelerating supersonically a magnetized plasma jet into vacuum. As the name itself suggests, these devices have a large resemblance with the traditional 'de Laval' nozzle, a convergent-divergent solid channel where a hot neutral is expanded to generate thrust. MNs are a technology belonging to the field of space electric propulsion[1, 2], a broad term to denote all rocket engines where the propellant is ionized into a plasma and accelerated to provide thrust using an external electric power source, in contrast to traditional space chemical propulsion, where the power for jet acceleration is provided by a combustion reaction of the propellant.

Since the early 70s, MNs have stirred a significant amount interest due to the many qualities that make them a superior device to produce thrust in space. Some of the more prominent advantages include the following.

- First, the handling of the plasma is fully contactless. This minimizes plasma-material interaction problems and reduces plasma losses, raising the efficiency of the device.

- Second, operation is also electrodeless. The present generation of plasma thrusters, like gridded ion thrusters and Hall effect thrusters, require some form of naked electrodes to accelerate the plasma or to neutralize the ion beam. In the $\mathrm{MN}$, ions and electrons are accelerated together into a current-free jet, without need for electrodes. This means a profound simplification at system level, with benefits on e.g. the complexity of the power processing and control unit and on the overall robustness of the system, as it removes one critical point of failure. This can become a key feature in the construction of high-durability systems that must withstand hundreds of thousands of hours of operation, far beyond current thruster capabilities.

- Third, since it is possible to tune the magnetic field shape and strength in-flight, the MN is highly adaptable to an ample continuum of operating points, facilitating the design of dual-mode plasma thrusters (able to run at high specific impulse, $I_{s p}$, and low thrust, $F$, and vice-versa) and systems with wide operational envelopes.

- Fourth, the MN can in principle operate under different propellants. Not only does this open a path to use alternative gases instead of the traditional (and expensive) xenon, when the MN is combined with the adequate plasma source, but it is also a promising feature for space missions that require refueling by reusing the available resources at destination. 


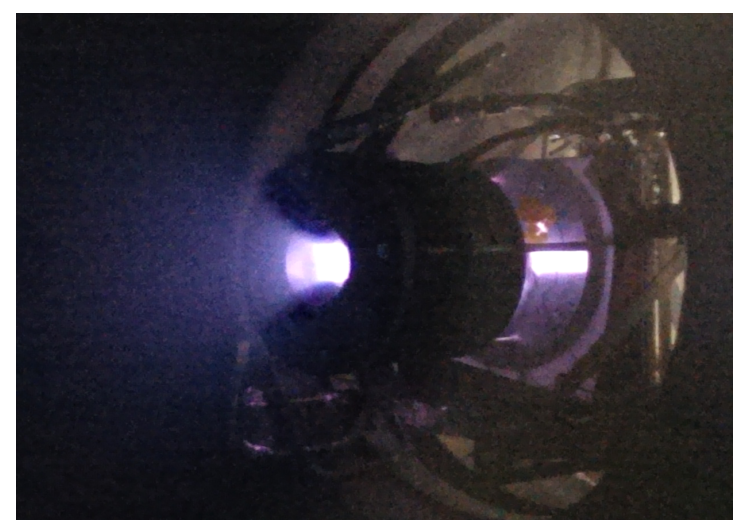

Figure 1: Magnetic nozzle of the helicon plasma thruster prototype HPT05 built by SENER Ingeniería y Sistemas and the EP2 research group during a recent test campaign at ESTEC, ESA, the Netherlands.

- Fifth, MNs enable the design of thrusters with a large thrust density, resulting in compact devices. This characteristic outperforms, in particular, the widespread gridded ion thrusters, where thrust density is limited by space charge effects between the grids.

- Lastly, the principles behind the MN are inherently scalable in power, size, and mass flow, covering a wide range of use cases, from hundreds of $\mathrm{W}$ to MW.

Due to all these advantages, MNs are regarded as a great candidate for mid/high-power electric propulsion $(>20 \mathrm{~kW})$, a propulsive niche that is gaining interest for both commercial telecommunication spacecraft (next generation satellite platforms are designed with high embarked power) and future human exploration missions.

Presently, MNs are being proposed as the acceleration stage of several nextgeneration space plasma thrusters, such as the Helicon Plasma Thruster $[3,4,5]$ (HPT), the Electron Cyclotron Resonance Thruster[6] (ECRT), the AppliedField MagnetoPlasmaDynamic thruster[7, 8, 9] (AF-MPDT), and the Variable Specific Impulse Rocket[10] (VASIMR). The HPT and ECRT both consist of a cylindrical plasma source that delivers RF power to the plasma, in order to sustain the discharge and energize it. The source typically ends at the throat of the MN, where the plasma is expanded into a high-velocity jet. The AFMPDT creates a DC discharge between two coaxial electrodes, and a quasi-axial magnetic field forms the MN. Finally, the VASIMR is very similar in conception to the HPT, but an additional intermediate stage of ion cyclotron resonance heating (ICRH) is included between the source and the MN to further energize the plasma. An example of the operation of the MN of a $1 \mathrm{~kW}$ HPT prototype running on argon can be seen in Fig. 1. 
Back on Earth, MN expansions also find their use in other fields such as advance material processing $[11,12]$, but the requirements differ from the propulsive application.

Current knowledge of MN physics has been achieved thanks to a limited number of experiments, normally carried out during the characterization of one of the aforementioned plasma thrusters, and theoretical models of plasma expansions in a magnetic field. As relevant examples, the experiments of Andersen et al.[13], Kuriki et al.[14], York et al.[15] and Inutake et al.[16] demonstrate the basic operation of the MN, including the supersonic acceleration of the ion flow. Simple fluid 1D models were the first to be formulated, followed by 2D singleparticle models and 2D fluid models, such as those of Kosmahl[17], Chubb[18], Raadu[19], Gerwin et al.[20], Sercel[21], Hooper[22], Mikellides et al.[23], and Ahedo and Merino[24, 25]. As understanding of the key physical phenomena advanced, the description of the MN has been gradually refined, and several incorrect assumptions identified and corrected.

In order for MNs to be usable for propulsive applications, the plasma jet must detach from the applied magnetic field downstream, which consists of closed magnetic lines, to form a free-expanding plasma plume. Since the inception of the MN, the plasma detachment process has been a major concern, as failure to detach the plasma would lead to an energetic backflow that would impinge and damage the delicate surfaces of the spacecraft, and to the cancellation of produced thrust. Detachment has therefore received a lot of attention from theoreticians and experimentalists alike: Experiments by Cox et al.[26], Deline et al.[27], Terasaka et al.[28], Squire et al.[29], Takahashi et al..[30] and Olsen et al.[31] all show that detachment begins to take place, to some extent, in the near-region of the expansion (i.e., up to a few plasma radii away from the thruster exit). Notwithstanding this, a clear explanation of this phenomenon has remained elusive for the last two decades. Moses et al.[32], Hooper[22] and Arefiev et al.[33] proposed different separation mechanisms, based on different types of MHD cold-plasma models. However, the conclusions of these studies were afterward shown to be inapplicable to MN expansion of hot plasmas, the case of interest for propulsion[25, 34]. Recently, Merino and Ahedo have explained plasma detachment as the gradual separation of ion streamtubes from the magnetic field due to ion inertia[35], as supersonic ions demagnetize and the ambipolar electric field is unable to keep them tied to magnetic streamtubes.

This review is primarily articulated around the work of our research group on MNs. It covers the fundamental physics of the MN, with an emphasis on the explanation of plasma acceleration, thrust generation, and detachment. The propulsive figures of merit of the device are investigated, describing the parametric region where the MN is expected to function more efficiently. The influence of several advanced physical mechanisms in the operation of the device is then assessed, and the state-of-the-art of MN modeling is discussed, offering suggestions for its advancement. An extensive reference list is included that encompasses most of the existing literature on MNs.

The rest of the paper is organized as follows. Section 2 establishes a two-fluid, two-dimensional model of the plasma expansion in a MN, and uses it to explore 
the fundamentals of ion acceleration, energy conversion, thrust generation, and plasma detachment. In Section 3, the performance of the MN (thrust gain, plume efficiency and plume divergence) is discussed. Section 4 gradually extends the basic model to explore other aspects of MN physics and their relevance in propulsive applications. Lastly, conclusions and closing remarks on open areas of research are gathered in Section 5 .

\section{Fundamental physics of magnetic nozzles}

In order to explore the physical principles behind the operation of the MN, a basic model of the plasma flow in a expanding magnetic field will be established first. The archetypal plasma used in MN-based electric thrusters is generated by a $\mathrm{DC}$ or $\mathrm{RF}$ discharge, providing electron densities ranging from $10^{16} \mathrm{~m}^{3}$ to about $10^{19} \mathrm{~m}^{3}$ and an electron temperature up to several tens of $\mathrm{eV}$. The plasma jet at the exit of the thruster has a radius that can go from less than 1 $\mathrm{cm}$ to about $10 \mathrm{~cm}$. Magnetic fields with strengths from $200 \mathrm{G}$ to $2 \mathrm{~T}$ are used, while the specifics of the generation and geometry of the field change substantially among the different thruster types. The materials commonly proposed as propellants include xenon, hydrogen, argon, lithium, mercury, or even iodine. Molecular gases such as $\mathrm{CO}_{2}$ and others are also being considered. Notwithstanding this, due to its heritage in electric propulsion and higher atomic mass, xenon continues to be one of the preferred propellants.

With such a wide range of parameters, it is difficult to describe the physics of all possible MN regimes in a simple way. Nonetheless, some general traits of MN plasma flows can be identified that are used to set up a general theoretical framework for their study. The discussion here focuses on the divergent part of the MN, i.e., the one that takes place outside of the ionization chamber of the thruster.

Firstly, plasma is in general sonically-chocked at the magnetic throat (the section where magnetic lines have minimal radius), and the expansion in the divergent $\mathrm{MN}$ is supersonic for the ions. Density decreases fast in the expansion and collisionality is low, except perhaps near the throat. Secondly, most plasma sources currently envisaged for propulsion deposit most of the internal energy on the electrons. A prominent exception is the VASIMR, in which the ICRH stage delivers a large fraction of the applied power to the ions directly. Thirdly, under the magnetic strengths and ion masses noted above, electrons can be considered well-magnetized in a large region of the MN (i.e., the electron Larmor radius is far smaller than the macroscopic scale of the problem), whereas ions are typically only partially magnetized near the $\mathrm{MN}$ throat where the field is maximum, if at all.

These three basic observations form the core assumptions of the two-fluid, two-dimensional plasma model first introduced in Ref. [24] and briefly described below. This model has been successful in explaining the dominant plasma physics in the $\mathrm{MN}$, and has been used as the basis for other models and works of other groups, such as in Ref. [36]. Afterward in this section the model is used to 


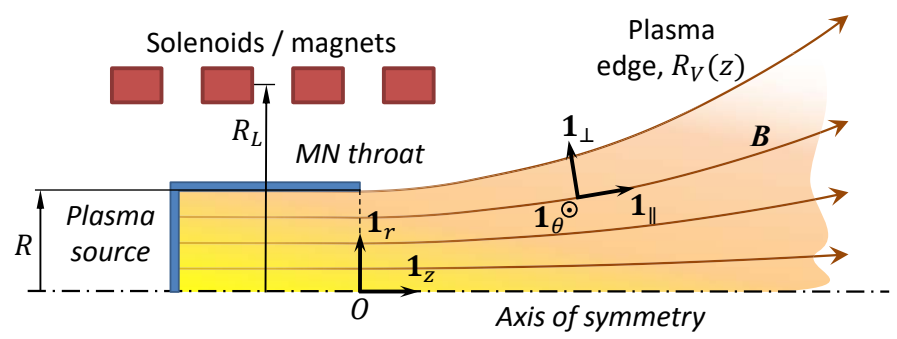

Figure 2: Sketch of a plasma jet flowing into a magnetic nozzle from a plasma source. The magnetic nozzle is created by a set of solenoids or permanent magnets of average radius $R_{L}$. The plasma throat with radius $R$, the origin of coordinates, the plasma edge $r=R_{V}(z)$ and the unit vectors $\mathbf{1}_{z}, \mathbf{1}_{r}, \mathbf{1}_{\theta}, \mathbf{1}_{\|}$, and $\mathbf{1}_{\perp}$ are shown for reference.

investigate the fundamental physics of the plasma expansion, thrust generation, and plasma detachment in the MN.

\subsection{Model of the plasma flow in a magnetic nozzle}

Consider the divergent, axisymmetric MN created by a set of coils, solenoids, and/or permanent magnets (Fig. 2). In order to express the different vector quantities of the MN, the cylindric reference frame $\left\{\mathbf{1}_{z}, \mathbf{1}_{r}, \mathbf{1}_{\theta}\right\}$ will be used. The origin of the reference frame is the center of the magnetic throat. Additionally, the following reference frame associated to the magnetic field $\boldsymbol{B}$ is introduced: $\left\{\mathbf{1}_{\|}, \mathbf{1}_{\perp}, \mathbf{1}_{\theta}\right\}$, with $\mathbf{1}_{\|}=B / B$ and $\mathbf{1}_{\perp}=\mathbf{1}_{\theta} \times \mathbf{1}_{\|}$.

The solenoidal magnetic field $\boldsymbol{B}$ can be expressed through its streamfunction $\psi$ as $\nabla \psi=r B \mathbf{1}_{\perp}$, i.e.,

$$
\partial \psi / \partial z=-r B_{r}, \quad \partial \psi / \partial r=r B_{z} .
$$

In general, $\boldsymbol{B}$ is given by the sum of the applied and the plasma-induced magnetic fields, $\boldsymbol{B}=\boldsymbol{B}_{a}+\boldsymbol{B}_{p}$; note that streamfunctions can be defined for each of these fields, $\psi_{a}$ and $\psi_{p}$, in a similar manner to $\psi$. Without loss of generality, it is assumed that the direction of the applied magnetic field is such that $B_{a z}(0,0)>0$.

A steady-state, fully-ionized plasma jet made of singly-charged ions of mass $m_{i}$ and electrons of mass $m_{e}$ enters the divergent $\mathrm{MN}$ at the throat with radius $R$, where plasma properties and radial profiles are known. This radius can be regarded as the macroscopic length of the problem. To proceed with the derivation of a simplified model that captures the main MN physics, the following hypotheses about the plasma are made; where applicable, a reference to the section that further discusses that hypothesis is provided:

1. The plasma is quasineutral, that is, the Debye length in the plasma $\lambda_{D}$ is much smaller than $R$. Then, electron and ion densities coincide everywhere: $n \equiv n_{e}=n_{i}$. 
2. At any instant of time, the same amount of ions and electrons flows out of the thruster and into the MN, to ensure that the thruster does not charge up electrically with time (global zero-current condition). Note that this does not preclude the existence of electric current locally within the plasma, and in fact it can be $\boldsymbol{j} \neq 0$ as long as its integral across any given section of the $\mathrm{MN}$ is zero. In particular, the azimuthal electric current $j_{\theta}$ is central in the operation of the MN, as it will be seen in Section 2.3. Local meridional (longitudinal) currents are also non-zero in general.

3. Electrons carry all the internal energy of the plasma, while ions are comparatively cold. Therefore, ion temperature and pressure will be neglected in the model (i.e, ion temperature $T_{i} \ll T_{e}$, the electron temperature). Other possibilities are explored in Section 4.2.

4. The induced field $\boldsymbol{B}_{p}$ is negligible with respect to $\boldsymbol{B}_{a}$, so that $\boldsymbol{B} \simeq \boldsymbol{B}_{a}$. Induced field effects will be investigated in Section 4.3. As shown there, the condition $B_{p} \ll B_{p}$ is equivalent to having a small plasma-beta parameter, $\beta=\mu_{0} n T_{e} / B_{a}^{2} \ll 1$, with $n T_{e}$ the electron pressure, and $\mu_{0}$ the magnetic permeability of free space.

5. The plasma is considered collisionless, i.e., the shortest collisional mean free path $\lambda_{c}$ is much larger than $R$. The deviation from this condition is investigated in Section 4.4.

6. Regarding the magnetization degree of each species, electrons will be considered fully magnetized in the whole $\mathrm{MN}$, under the assumption that their Larmor radius verifies $\ell_{e} \ll R$, or, what is the same, that their gyrofrequency $\Omega_{e}$ satisfies $\Omega_{e} \gg \sqrt{T_{e} / m_{e}} / R$, where $m_{e}$ is the electron mass. Note that full electron magnetization also requires the Hall parameter to be $\chi_{H}=\Omega_{e} / \nu_{e} \gg 1$, with $\nu_{e}$ the total electron collision frequency. This condition, already satisfied by virtue of hypothesis number 5 , ensures that collisional processes do not disturb substantially the electron gyromotion and that cross-field diffusion is negligible.

On the other hand, ions are allowed to have any degree of magnetization, and they are commonly only partially magnetized. Therefore, the distinguished limit $\Omega_{i} \lesssim u_{i} / R$ (with $\Omega_{i}$ the ion gyrofrequencey and $u_{i}$ the ion velocity) is adopted in the model.

7. Electron inertia is neglected, which basically relies on $m_{e} \ll m_{i}$. A detailed discussion of electron inertia effects can be found in Section 4.5.

Since the interest of this discussion lies primarily in the macroscopic, bulk behavior of the plasma expansion in the $\mathrm{MN}$, and not in the motion of individual particles or the detailed distribution functions, each species will be modeled as a fluid. Under the prior assumptions, the steady-state, two-fluid plasma obeys 
the following continuity and momentum equations:

$$
\begin{aligned}
\nabla \cdot\left(n \boldsymbol{u}_{i}\right) & =0 ; \\
\nabla \cdot\left(n \boldsymbol{u}_{e}\right) & =0 ; \\
m_{i}\left(\boldsymbol{u}_{i} \cdot \nabla\right) \boldsymbol{u}_{i} & =-e \nabla \phi+e \boldsymbol{u}_{i} \times \boldsymbol{B} ; \\
0 & =-\nabla \cdot \mathcal{P}_{e}+e n \nabla \phi-e n u_{\theta e} B \mathbf{1}_{\perp},
\end{aligned}
$$

with $e$ the electron charge, $\phi$ the electric potential, and $\mathcal{P}_{e}$ the pressure tensor of electrons. Observe that the continuity equation for each species allows defining a couple of streamfunctions that satisfy:

$$
\frac{\partial \psi_{j}}{\partial z}=-r n u_{r j} ; \quad \frac{\partial \psi_{j}}{\partial r}=r n u_{z j},
$$

with $j=i, e$ for ions and electrons, respectively.

The motion of fully-magnetized electrons (hypothesis 6) faithfully follows the magnetic field, except for the azimuthal drifts that may exist in the MN (for example, the $E \times B$ and diamagnetic drifts), and therefore electron streamtubes coincide with magnetic streamtubes. In other words, electron velocity can be written as:

$$
\begin{aligned}
\boldsymbol{u}_{e} & =u_{\| e} \mathbf{1}_{\|}+u_{\theta e} \mathbf{1}_{\theta}, \\
u_{\perp e} & \equiv 0 .
\end{aligned}
$$

This allows the simplification of Eq. (3), which thanks to the solenoidal nature of $\boldsymbol{B}$ can be written as

$$
n u_{\| e} / B=G_{e}(\psi),
$$

where $G_{e}(\psi)$ is the electron-to-magnetic flux ratio, which can be computed for each electron tube from the initial conditions at the throat. By virtue of quasineutrality and full electron magnetization, the plasma is bounded by the outermost magnetic tube that departs from the plasma source exit section, after which there is a clean transition into vacuum. The radius of this magnetic tube therefore defines the radius of the plasma jet edge, $R_{V}(z)$ [with $R_{V}(0)=R$ ].

It is worth noting that, within the assumptions listed above, these fluid equations are exact and follow directly from the integration of the moments of Vlasov's equation for each species. Nonetheless, as with every fluid model, a closure for the highest moment of the distribution kept - in the present case, $\mathcal{P}_{e}$, - is required. While the correct closure for $\mathcal{P}_{e}$ must come from a kinetic description of the electrons, for simplicity a Maxwellian, isotropic species of temperature $T_{e}$ is assumed. Moreover, due to the ambipolar electric field that develops, electrons are a nearly-confined species, where only a small high-energy tail of the population actually flows downstream with the ions in order to fulfill the zero-current condition. As such, in a first approach, the high-mobility electrons are further considered to be isothermal, i.e. $T_{e}=T_{e 0}=$ const, where subindex 0 denotes the value at the origin. Other descriptions of the electron population are considered in Section 4.1. 
Under this description of the electron species, electron pressure is a scalar, i.e. $p_{e}=n T_{e 0}$, and Eq. (5) simplifies to:

$$
0=-T_{e 0} \nabla \ln \left(n / n_{0}\right)+e \nabla \phi-e u_{\theta e} B \mathbf{1}_{\perp} .
$$

Observe that Eq. (5) [or Eq. (10)] has only two useful scalar components in the axisymmetric $\mathrm{MN}$, as the component along $\mathbf{1}_{\theta}$ has been substituted by the condition $u_{\perp e} \equiv 0$.

The model composed by Eqs. (2), (4), (9) and (10) (a total of 7 scalar equations) encloses the dominant physics of the supersonic expansion of a plasma in the MN. Given the magnetic field $\boldsymbol{B}$ and a set of valid initial conditions, the model can be solved for $n, \phi, u_{\| e}, u_{\theta e}$ and $\boldsymbol{u}_{i}$. Assuming that the density profile at the throat is $n=n(0, r)(0<r<R)$, one possible choice of initial conditions, which sensibly represents existing MN thrusters such as the HPT is given by:

$$
\begin{aligned}
\phi(0, r) & =0, & \int_{0}^{R}\left(n u_{z i}-n u_{z e}\right) r d r & =0, \\
u_{z i}(0, r) & =c_{s} \equiv \sqrt{\frac{T_{e}}{m_{i}}}, & u_{r i}(0, r)=u_{r e}(0, r) & =0, \\
u_{\theta e}(0, r) & =-\frac{T_{e 0}}{e B} \frac{\partial \ln \left(n / n_{0}\right)}{\partial r}, & u_{\theta i}(0, r) & =0 .
\end{aligned}
$$

The conditions in Eq.(11) impose that there is no initial radial electric field at the throat and that the total electric current leaving the thruster is zero, consistent with Ref. [37]. Eq.(12) states that the flow at the throat is axial and that ions are sonically chocked, i.e. their velocity equals the plasma sonic velocity, $c_{s}$. This last condition is equivalent to the (longitudinal) ion Mach number, $M=\sqrt{u_{z i}^{2}+u_{r i}^{2}} / c_{s}$, being equal to 1 . Moreover, this condition means that the ion mass flow through the MN is fixed and equal to $\dot{m}_{i}=\pi R^{2} \bar{n} c_{s}$, with $\bar{n}$ the average plasma density at the throat.

Finally, the two expressions in Eq. (13) mean that electrons need to be in a $\theta$-pinch equilibrium [38] to ensure the radial equilibrium of forces at the throat, with $e u_{\theta e} B \sim T_{e 0} / R$, and that the plasma has no initial rotation, respectively.

Normalizing the equations with $R, m_{i}, e, T_{e 0}$ and $n_{0}=n(0,0)$, the model is seen to depend only on the following.

1. The geometry of the applied field, and more particularly, its divergence rate. In the simplest case that the applied field is generated by a single loop of current of radius $R_{L}$ (with $R_{L}>R$ ) located at $z=0$, the geometry is defined just by the parameter $R_{L} / R$.

2. The throat profile functions $n(0, r)$ and $u_{z e}(0, r)$.

3. The relative strength of the magnetic field, easily discussed with the nondimensional ion gyrofrequency parameter, $\hat{\Omega}_{i 0}=e B_{0} R /\left(m_{i} c_{s}\right)$.

Out of these three dependencies, $\hat{\Omega}_{i 0}$ is the most relevant for the operating regime of the MN. In the following, whenever dimensionless quantities are more appropriate for the discussion, a hat will be used to denote them: e.g., $\hat{\phi}=$ $e \phi / T_{e 0}, \hat{n}=n / n_{0}$, etc. 


\subsection{Electron fluid and supersonic expansion of ions}

The interplay between the magnetized electrons and the cold ions is of utmost importance for the operation of a MN. Without need of integrating the model, it is possible to already advance some of the key properties of the electron flow.

First, observe that $u_{\| e}$ is only involved in Eq. (9), and that it is indeed decoupled from the rest of the problem. One may, therefore, use this equation to obtain $u_{\| e}$ after solving for the other 6 variables of the problem, once $n$ is known. Physically, this means that the longitudinal electron current doest not affect the ion expansion to first-order. Second, closer inspection of Eq. (10) reveals that the electron density follows a Boltzmann relation along each magnetic line. This becomes apparent after projecting Eq. (10) along $\mathbf{1}_{\|}$,

$$
T_{e 0} \ln \left(n / n_{0}\right)-e \phi=H_{e}(\psi) .
$$

This Bernoulli-like equation states that there is a univocal relation between plasma density and plasma potential on each electron/magnetic tube, given by the function $H_{e}(\psi)$, which can be computed for each electron tube from the throat conditions. Observe that $-H_{e} / e$ is sometimes called the 'thermalized potential.'

Using this last expression, the projection of Eq. (10) along $\mathbf{1}_{\perp}$ yields the value of the electron azimuthal velocity:

$$
u_{\theta e}=\frac{1}{e B}\left(e \frac{\partial \phi}{\partial \mathbf{1}_{\perp}}-T_{e 0} \frac{\partial \ln \left(n / n_{0}\right)}{\partial \mathbf{1}_{\perp}}\right)=-\frac{1}{e B} \frac{\partial H_{e}}{\partial \mathbf{1}_{\perp}}=-\frac{r}{e} \frac{d H_{e}}{d \psi} .
$$

As it can be inferred from the first equality in Eq. (15), $u_{\theta e}$ results from the summation of the $E \times B$ drift and the diamagnetic drift. Nonetheless, this sum is given solely by the initial conditions, and can be known for each electron tube before solving the expansion in the divergent MN. In other words, at each point $u_{\theta e}$ is the necessary one to make the magnetic force term in Eq. (10) compensate the imbalance between the outward-pointing electron pressure and the inwardpointing electric force on electrons; and this imbalance in the $\mathrm{MN}$ is already dictated by the profile of $d H_{e} / d \psi$ at the throat. One last observation about Eq. (15) is that the azimuthal angular velocity, $u_{\theta e} / r$, is also a function of $\psi$ only; in other contexts, this behavior has been referred to as the isorotation of the electron flow $[39,34]$.

The conservation of $G_{e}, H_{e}$ and $u_{\theta e} / r$ along electron/magnetic streamtubes constitutes a set of three first-integrals of the electron equations of the model, which therefore become fully algebraic.

The remaining four ion equations can also be worked out to bear some additional insight. Firstly, the projection of the momentum equation Eq. (4) along $\boldsymbol{u}_{i}$ leads to the conservation of ion mechanical energy, $H_{i}$ along ion streamlines,

$$
\frac{1}{2} m_{i} u_{i}^{2}+e \phi=H_{i}\left(\psi_{i}\right) .
$$

Secondly, an additional first-integral may be obtained from the projection of Eq. (4) along $\mathbf{1}_{\theta}$, which yields the conservation of canonical angular momentum 
of ions, $D_{i}$, on each ion streamline:

$$
r m_{i} u_{\theta i}+e \psi=D_{i}\left(\psi_{i}\right) .
$$

To sum up, the number of partial differential equations of the model boils down to 2. Since the ion flow is supersonic, these last two equations constitute a hyperbolic system that can be readily solved numerically using, for example, the method of characteristics[40]. This is indeed the choice followed in the DIMAGNO code introduced in Ref. [24].

The two-dimensional plasma expansion is illustrated in Fig. 3 for a representative MN flow and several ion magnetization degrees, described by the parameter $\hat{\Omega}_{i 0}$. These results confirm that ion acceleration is taking place as density (and the ambipolar electric potential) decrease. The behavior of the ion Mach number agrees with the one observed experimentally[13, 16].

The $2 \mathrm{D}$ character of the expansion becomes more marked as the flow advances, where the ion Mach number, density, and potential at the axis $(r=0)$ and at the plasma-vacuum transition line $\left[r=R_{V}(z)\right]$ begin to differ. Rarefaction and acceleration at a given axial position $z$ are more pronounced at the plasma periphery. The effect is clearly observed in Fig. 3( $d)$, where the density profile is plotted for different values of $z$. Here, one can appreciate the relative focalization of the density, which does not expand in a self-similar way; quite on the contrary, it is seen how the peripheral plasma becomes tenuous faster than the plasma near the axis. As it will be seen in Section 2.4, this phenomenon is largely beneficial for plasma detachment and to keep a moderate plume divergence angle.

Indeed, this focalization is linked to the gradual inward separation of the ion streamlines from the magnetic field represented on Fig. 4. The fact that electrons are fully magnetized in the $\mathrm{MN}$ while ions are not means that the latter do not necessary follow the same trajectories as the former, and indeed initially-coinciding ion and electron streamtubes separate with respect to each other. This gives rise to local electric currents in the meridional plane, $j_{z}$ and $j_{r}$, even in a globally current free, quasineutral plasma. Actually, the magnitude of these currents can become comparable to the ion current itself downstream[24].

In regard with the role of the ion magnetization degree $\hat{\Omega}_{i 0}$, the plasma responses for $\hat{\Omega}_{i 0}=0.1$ (case with nearly-unmagnetized ions everywhere) and 100 (case with highly-magnetized ions at the throat) are shown in Fig. 3 as different lines. Clearly, the effect of this parameter is nearly irrelevant for $M$, $n$ and $\phi$ in this range; the only noticeable effect of a higher $\hat{\Omega}_{i 0}$ in these graphs is a lower degree of radial relative density focalization. Additional simulations confirm that these results are nearly-coincident with the $\hat{\Omega}_{i 0} \rightarrow 0$ limit, where the magnetic force on ions is neglected. The reason lies in that the magnetic field decreases fast (as $\hat{R}_{V}^{-2}$ ), while the accelerating ions are gaining velocity; this means that the ion magnetic force soon becomes irrelevant compared to the ion inertial term in Eq. (2). Only at very large ion magnetization strengths, far higher than those envisaged for practical applications, does the ion flow remain sufficiently magnetized downstream and the magnetic force on ions is 
$(a)$

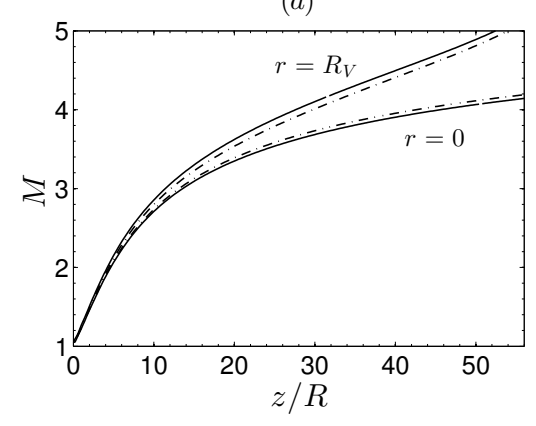

(b)

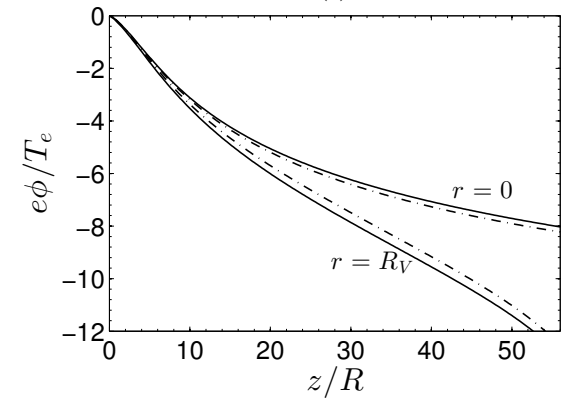

(c)

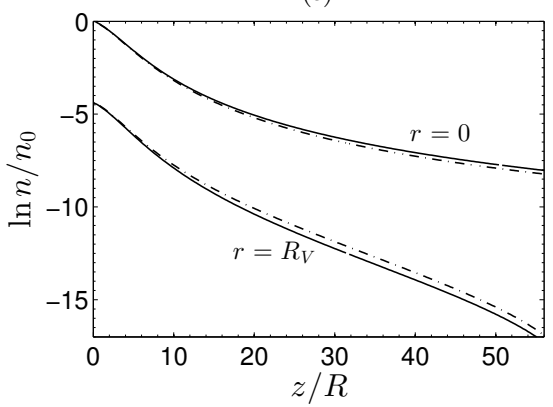

(d)

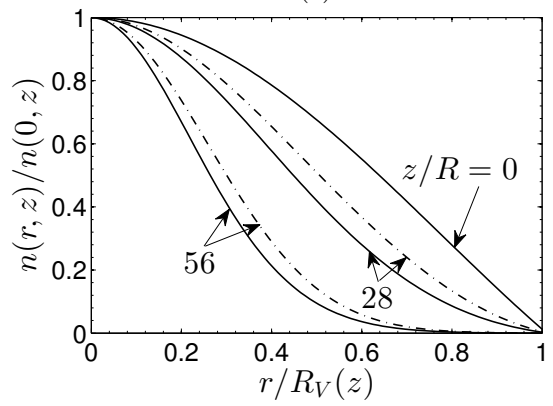

Figure 3: Evolution along the axis $(r=0)$ and the plasma edge $\left(r=R_{V}\right)$ of the ion Mach number $M(a)$, the electric potential $\phi(b)$, and the plasma density $n(c)$ in a MN generated by a single coil of radius $R_{L}=5.4 R$ and a non-uniform initial plasma profile based on the $J_{0}$ Bessel function. In $(d)$ the radial density profile at three different values of $z$ has been plotted, normalizing the abscissa with $R_{V}(z)$. Solid lines correspond to $\hat{\Omega}_{i 0}=0.1$, and dash-dot lines to $\hat{\Omega}_{i 0}=100$, a range that amply covers foreseen applications. Source: Adapted from Ref. [24]. (c) 2010 AIP. Adapted with permission. 


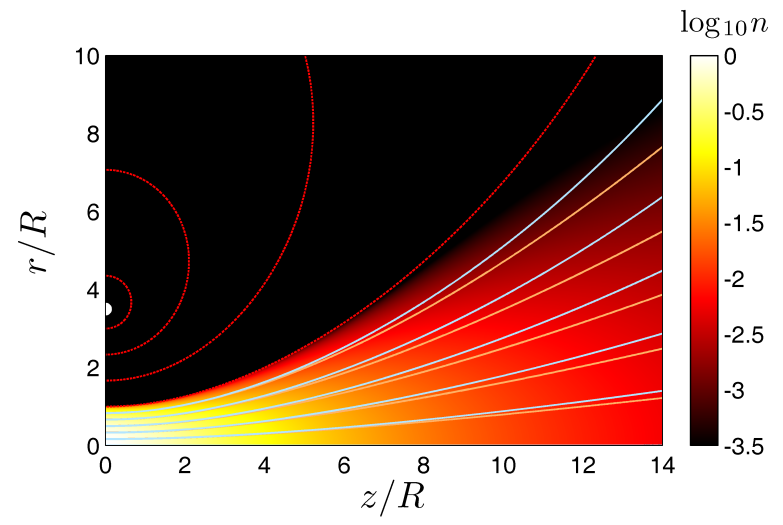

Figure 4: Ion and electron/magnetic streamtubes in the MN (orange and blue, respectively) for a $\mathrm{MN}$ generated by a single coil of radius $R_{L}=3.5 R$ and a nonuniform initial plasma profile based on the $J_{0}$ Bessel function (see Fig. 3). Each pair of ion and electron streamtubes coincides initially at the MN throat. Additional magnetic lines outside of the plasma are shown in red. The background color map shows the $2 \mathrm{D}$ plasma density in the expansion.

large enough to observe noticeable differences in the focalization phenomenon and in ion separation.

As a side note, observe that $1 \mathrm{D}$ models miss most of these phenomena, and are only adequate to provide averaged values of the plasma acceleration and density[24]. They also conceal the azimuthal velocities $u_{\theta e}$ and $u_{\theta i}$, which are a central feature of the expansion, as discussed further below.

\subsection{Energy conversion and thrust generation}

As already anticipated by Eq. (16), ion acceleration is afforded by the drop in the ambipolar electric potential $\phi$. As electrons are hot and have high mobility, they tend to rush ahead of ions while they follow the magnetic tubes. In doing so, a small space-charge and an ambipolar electric field forms which confines most of the electrons upstream and accelerates the ions downstream. In the present mathematical model, where the quasineutrality approximation is imposed, this electric field is set up by the electron force balance [Eq. (14)].

Thanks to the ambipolar electric field, the internal energy of electrons is transformed into directed kinetic energy of ions. For the MN and plasma types being considered, this is the main ion acceleration mechanism. Since $\phi$ scales with $T_{e 0}$ [Eq. (14)], having a high ion acceleration requires a high value of $T_{e 0}$.

This ambipolar conversion mechanism, however, is a common feature of all plasma expansions into vacuum, magnetized or otherwise[41]; the main benefit of a $\mathrm{MN}$ is that most of the perpendicular internal energy of electrons is not wasted uselessly into accelerating the plasma in the radial direction, as in the 
case of an unmagnetized plasma. Instead, the radial expansion is confined by the magnetic force that results from the interaction between the applied magnetic field and the electric currents in the plasma. Mirroring the behavior of a solid de Laval nozzle with a neutral hot gas, where the divergent walls help to transform the radial energy and momentum of the gas into additional axial energy and momentum to increase thrust, the magnetic forces in the MN have a radial and an axial component, and gradually convert the electron perpendicular internal energy and momentum into parallel energy and momentum. The guiding magnetic field therefore helps to obtain a more directed expansion and to take advantage of the perpendicular internal energy of electrons.

While ions are accelerated by the ambipolar electric field, it still remains to be discussed how thrust is transmitted back to the MN. Thrust is defined as the forward force felt by the thruster when it is in operation. Obviously, by momentum conservation, this force must equal the amount of axial plasma momentum leaving the system (thruster plus MN) per unit of time. The thrust force is composed of two contributions. The first one is the internal thrust $F_{0}$, associated to the acceleration of the plasma within the thruster chamber as it reaches sonic conditions at the $\mathrm{MN}$ throat. In most cases, this thrust is mainly mechanical due to plasma pressure on wall chambers. The second contribution is related to the thrust gain in the divergent $\mathrm{MN}$, and is referred to as magnetic thrust. This contribution is associated to the increase of total axial momentum in the plasma as it expands supersonically in the MN, and results from the magnetic interaction between the magnetic circuit of the thruster and the azimuthal electric currents that exist in the plasma. Indeed, in the same way as the applied magnetic field creates a magnetic force on the plasma currents that confines and pushes the plasma, the plasma currents create an induced field that causes a reaction magnetic force on the thruster coils and magnets, equal and opposite to the former, of which its axial component is the magnetic thrust (see Fig. 5(a)). Observe that, while the plasma-induced field may not be strong enough to modify the MN field and the plasma expansion (see Section 4.3), the reaction force it creates on the large azimuthal currents of the magnetic generator is not negligible.

The axial magnetic force density on the plasma is shown for a representative simulation in Fig.6. Visibly, it is in the near-region of the expansion, where plasma density and currents are large and the applied magnetic field starts to diverge but is still strong enough, where the largest contribution to magnetic thrust is produced. After the maximum contribution point, the radiallyintegrated force decreases slowly downstream. A recent experiment by Takahashi et al. has managed to measure the two thrust contributions, internal and magnetic, independently[42].

From this discussion, it is apparent that azimuthal plasma currents play a fundamental role in the plasma expansion and thrust generation. An important feature standing out from the model is that the electron and ion azimuthal currents respond to very different mechanisms: the diamagnetic and $E \times B$ drift origin of $u_{\theta e}$ and its downstream behavior have already been commented on. In a hot electron plasma the electron Hall current, $j_{\theta e}=-e n u_{\theta e}$ is the dominant 


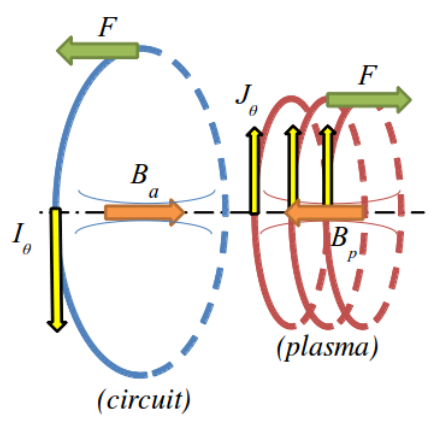

(a)

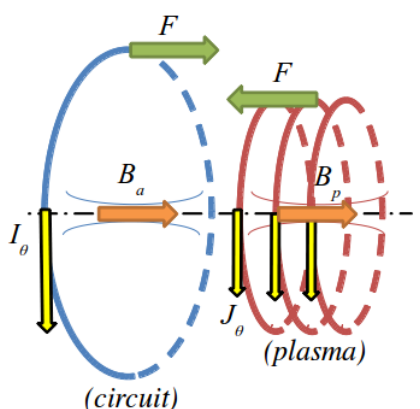

(b)

Figure 5: Sketch of the electric current $I_{\theta}$ on the magnetic circuit of the plasma (blue circles) and the azimuthal electric currents $j_{\theta}$ in the plasma (red circles). For $B_{z}(0,0)>0$, the current in the $\mathrm{MN}$ loop is positive along $\theta$. In the sketch $(a)$, circuit and plasma currents oppose each other, and therefore a repulsive magnetic force appears that generates positive thrust (diamagnetic plasma case). In the sketch (b), circuit and plasma currents flow in the same direction, and an attractive force appears that generates drag or negative thrust (paramagnetic plasma case). Source: Adapted from Ref. [25]. (C) 2011 AIP. Adapted with permission.

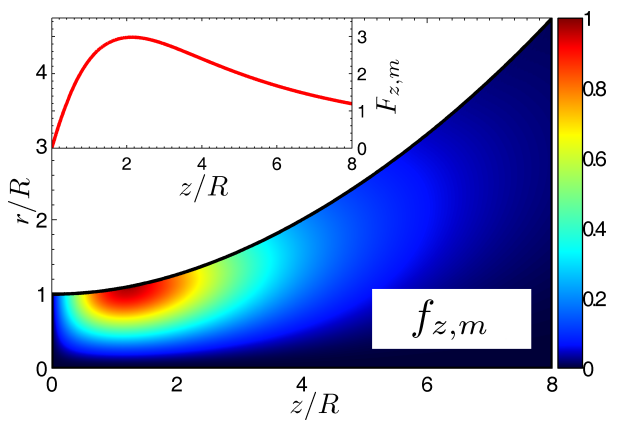

Figure 6: Magnetic thrust force density $f_{z, m}=-j_{\theta} B_{r}$ in a MN with $R_{L} / R=$ 3.5 and a non-uniform initial plasma profile based on the $J_{0}$ Bessel function. The color scale has been normalized with the maximum force density. In the upper sub-graph, the radially-integrated magnetic thrust force $F_{z, m}=\int_{0}^{R_{V}(z)} 2 \pi r f_{z, m} d r$ is plotted against $z$. Source: Adapted from Ref. [43]. (C) 2011 IEEE. Adapted with permission. 
azimuthal current contribution, and assuming without loss of generality that $B_{z}(0,0)>0, j_{\theta e}$ has a negative sign [Eq. (15)].

The ion azimuthal current, on the other hand, responds to the conservation of the canonical angular momentum. Even if ions are introduced without initial rotation into the $\mathrm{MN}$, they may develop a (small) azimuthal ion swirl current, $j_{\theta i}=e n u_{\theta i}$. The sign and magnitude of $u_{\theta i}$ and $j_{\theta i}$ become apparent from the study of Eq. (17). Recalling that ions separate inward from the magnetic field lines, i.e., toward lower values of $\psi$, a positive $j_{\theta i}$ develops downstream when $B_{z}(0,0)>0$; the magnitude of $u_{\theta i}$ is governed by the extent of the separation and the intensity of the magnetic field.

It is the competition of these two azimuthal currents of different sign that eventually defines the magnetic thrust. In a simplified way, the azimuthal plasma currents in the plasma can be regarded as distributed current loops of a given radius and intensity, interacting magnetically with the current loop that generates the MN, as was sketched in Fig. 5. As it is well known, the magnetic force between two current loops is repulsive when the two currents have opposite signs (diamagnetic currents), and attractive when their signs are the same (paramagnetic currents). Clearly, it is the first situation which provides a positive magnetic thrust force. With the aforementioned sign of $j_{\theta e}$ and $j_{\theta i}$ in the MN, the diamagnetic electron current creates positive thrust, whereas the paramagnetic ion current is responsible for a drag contribution, or negative thrust.

A quick calculation allows to define a set of sufficient conditions to have a positive axial magnetic force density on the plasma, i.e, a net diamagnetic current $\left(j_{\theta e}+j_{\theta i}\right) B_{r}<0$, or, what is the same, $\left(u_{\theta e}-u_{\theta i}\right)>0$. Again, it is assumed that $B_{z}(0,0)>0$, and the focus is on initially rotation-free plasmas. On the one hand, from Eq. (13) $u_{\theta e}>0$ for negative $d H_{e} / d \psi$, and it grows downstream with $r$ along each magnetic tube. On the other hand, from Eq. (17), it is easy to find an upper bound for $u_{\theta i}$ : for any given ion streamline, the largest ion azimuthal velocity would occur in the case of perfect separation from the magnetic lines, i.e., if the radius of the streamline remained constant and equal to the initial one, $r_{i}=r_{i 0}$, far downstream, as $\psi \rightarrow 0$. One can therefore write $u_{\theta i} \leq D_{i}\left(\psi_{i}\right) /\left(r_{i 0} m_{i}\right)$ at each ion tube. Since $u_{\theta i}=0$ initially in a rotation-free plasma, $D_{i}\left(\psi_{i}\right)=e \psi\left(0, r_{i 0}\right)$. Lastly, from the definition of $\psi$ [Eq. (1)],

$$
u_{\theta i}<\frac{e B\left(0, r_{i 0}\right) r_{i 0}}{2 m_{i}}<\frac{e B(0, R) R}{2 m_{i}} .
$$

Therefore, one may write the positive thrust density condition as

$$
-\left.\frac{\partial H_{e}}{\partial r}\right|_{\text {throat }}>\frac{e^{2} B^{2}(0, R) R}{2 m_{i}} .
$$

Taking orders of magnitude, the condition in Eq. (19) reads simply as $\hat{\Omega}_{i 0}^{2} \lesssim 1$. This expression provides the valuable indication that magnetic thrust density will be positive for low ion magnetization degrees, but may become negative at high values of the magnetic field or low electron temperatures. 
Note, however, that this estimation is extremely conservative due to the exigent bound that was chosen for $u_{\theta i}$. Actually, simulations show that for $\hat{\Omega}_{i 0}=$ 200 there is still not enough drag to cancel the produced thrust; moreover, even in the limit of $\hat{\Omega}_{i 0} \rightarrow \infty$, a positive thrust gain occurs initially in the near region of the MN: only farther downstream, about the region where magnetic lines have acquired a large radial divergence and start to turn back, paramagnetic ion drag becomes important, and may cancel out the magnetic thrust generated by the near region. This drag can be understood as the a strong attractive magnetic force on ions when $\hat{\Omega}_{i 0}$ is large, which forces them to follow the magnetic tubes and therefore makes them change axial momentum to radial momentum near the turning point.

The drag effect in high- $\hat{\Omega}_{i 0}$ plasmas becomes even more dramatic if ions are injected into the MN well above their sonic velocity, with $M_{0} \gg 1$. In this case, one speaks of an initially hypersonic plasma, or equivalently, a cold plasma, since $T_{e}$ is negligible compared to $m_{i} u_{i}^{2}$. The ambipolar electric field, which scales with $T_{e}$, is weaker with respect to ion inertia now, and therefore axial acceleration becomes less important. Same do the diamagnetic electron current and the thrust force density, which also scale with $T_{e}$. For the same reason, the ion paramagnetic force begins to dominate the expansion of the ion flow. As a result, the relative importance of ion drag grows.

In fact, in the $T_{e 0} \rightarrow 0$ limit (or for initial ion Mach number $M_{0} \rightarrow \infty$ ), the $\mathrm{MN}$ only provides drag, and therefore it is no longer useful for propulsion. This is consistent with the ambipolar energy conversion mechanism described above, which indicates that internal energy is required to sustain the ion acceleration and provide positive thrust. Hence, a cold plasma model cannot reproduce the generation of thrust in a MN.

\subsection{Plasma detachment from the magnetic field}

The incipient unmagnetized-ion separation observed in the near-region (Section 2.2) continues to develop in the far-region of the MN after the turning point of the last magnetic line (corresponding to $R_{V}$ ), and provides the natural answer to the plasma detachment issue[35]. Figure 7(a) displays the ion streamtubes of a simulation extending well into the far-region, showing that eventually their curvature tends to zero asymptotically and that separation grows unboundedly; only a tiny fraction of the plasma does actually turn around to accompany the magnetized electrons and keep the quasineutrality of the model. The bulk of the ions effectively detach from the magnetic field, forming a freely-expanding plasma plume. This behavior agrees well with the observed ion current separation in several MN experiments[26, 27, 28, 29, 30, 31], supporting ion separation due to demagnetization as the main detachment mechanism in the MN.

The cause of this ion separation can be easily understood from a simple orderof-magnitude calculation with the ion forces. Let us first define the directions $\mathbf{1}_{\| i}=\boldsymbol{u}_{i} / u_{i}$ and $\mathbf{1}_{\perp i}=\mathbf{1}_{\theta} \times \mathbf{1}_{\| i}$ associated to the ion flow. The relevant equation to understand ion deflection in the meridional plane is the projection of Eq. (4) 

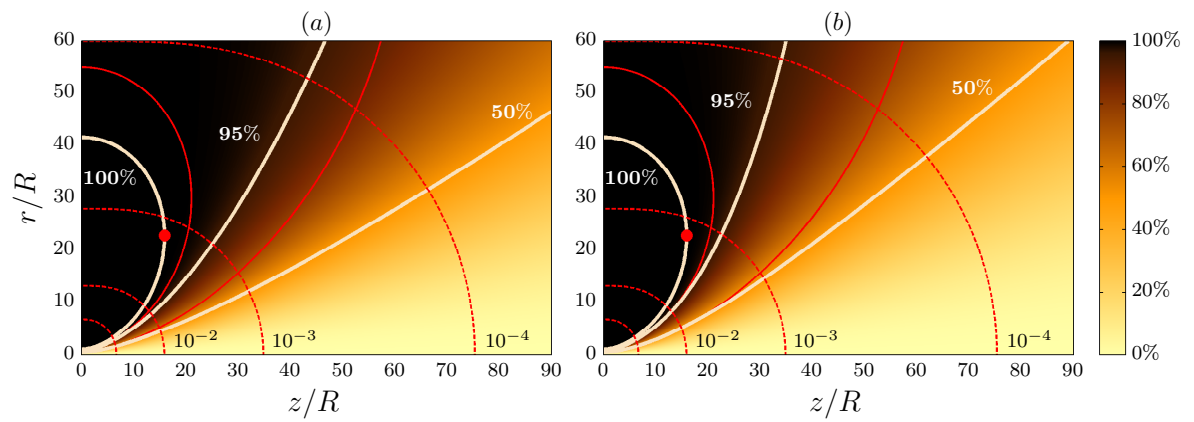

Figure 7: Far-region inward detachment of ions for two magnetic strengths: (a) $\hat{\Omega}_{i 0}=1$ and (b) 200, for a MN with $R_{L} / R=3.5$ and a non-uniform initial plasma profile based on the $J_{0}$ Bessel function. The ion streamtubes containing $50 \%, 95 \%$, and $100 \%$ of the plasma mass flow are shown as thick yellow lines. The initially-corresponding magnetic streamtubes are shown in solid thin red lines. The $2 \mathrm{D}$ map shows the integrated mass flow ( $0 \%$ on the axis, $100 \%$ on the beam edge). Dashed red lines are $B / B_{0}=$ const lines. The red dot marks the MN 'turning point.' Source: Adapted from Ref. [35]. (c) 2014 IOP. Adapted with permission.

along $\mathbf{1}_{\perp i}$, i.e.,

$$
m_{i} \kappa_{i} u_{i}^{2}=-e \frac{\partial \phi}{\partial \mathbf{1}_{\perp i}}+e u_{\theta i} B\left(\mathbf{1}_{\perp} \cdot \mathbf{1}_{\perp i}\right)+m_{i} \frac{u_{\theta i}^{2}}{r}\left(\mathbf{1}_{r} \cdot \mathbf{1}_{\perp i}\right),
$$

where $\kappa_{i}$ is the meridional curvature of ion streamtubes.

Eq. (20) provides an estimate of ion streamtube curvature in the bulk of the MN. Neglecting the small centrifugal term, and recalling that the electric field scales as $\sim T_{e 0} /(e R)$ and magnetic field as $\sim B_{0} R_{V}^{-2}$, we may write:

$$
\kappa_{i} R \sim \frac{1}{M^{2}}+\frac{\hat{\Omega}_{i 0}^{2}\left(B / B_{0}\right)}{M^{2}},
$$

where the order of magnitude of $u_{\theta i}$ has been calculated with the same conservative bound as before, Eq. (18).

From this analysis, it is apparent that $\hat{\Omega}_{i 0}$ is the relevant quantity to assess plasma detachment and the location where it takes place. At low values of $\hat{\Omega}_{i 0}$, ion magnetic force is negligible already from the beginning of the expansion, and $\kappa_{i} R \sim 1 / M^{2}$ in the bulk of the plasma. Therefore, as ions accelerate and $M$ increases, their trajectories straight out and soon become nearly-conic. A higher magnetization of ions, as could be expected, leads to a delayed ion separation and a higher divergence angle of the detached ion lines. Notwithstanding this, the second term in the right hand side of expression (21) decreases fast with increasing $M$ and decreasing $B / B_{0}$, the ratio of the local magnetic field to its value at the throat. In summary, as ions are accelerated and gain inertia, electric 
and magnetic forces eventually become insufficient to continue to deflect them radially to meet the magnetic lines, and as a necessary consequence, $\kappa_{i} \rightarrow 0$ when the ratio of these forces to ion inertia goes to zero[35].

In fact, in order to postpone ion detachment until e.g. the turning point of the nozzle, a very high ion magnetization degree is required: for the simulations shown in Fig. $7, B$ has already dropped 3 orders of magnitude at the MN turning point, and $M \simeq 5$ around that location; therefore, $\hat{\Omega}_{i 0} \gtrsim 2 \cdot 10^{2}$ is needed to maintain ion magnetization high enough for ions to remain attached at that position, and much more to keep it farther downstream. This is in agreement with Fig. $7(b)$, where ion separation is visibly smaller at the turning point section. For $T_{e 0}=10 \mathrm{eV}$, and $R=5 \mathrm{~cm}$, this translates into a magnetic intensity of at least $\sim 1.5 \mathrm{~T}$ for hydrogen and $\sim 15 \mathrm{~T}$ for xenon at the throat, far above the values considered practical for most thrusters.

Hence, ion separation will take place efficiently, ensuring the adequate detachment of the plasma mass and momentum from the $\mathrm{MN}$ and the formation of a free plasma plume. Moreover, there is a wide range of magnetic field strengths on which the MN can operate without detachment being substantially affected.

Recall that this solution is obtained under the conditions of zero electric net current, fully magnetized electrons, and quasineutrality everywhere. Naturally, and as already pointed out in Section 2.2, this does not preclude the formation of local meridional currents, which in fact are the natural consequence of ions separating from the electron/magnetic streamtubes. Observe that the charge conservation equation $\nabla \cdot \boldsymbol{j}=0$ [the combination Eqs. (2) and (3)] does not imply the local current ambipolarity condition in the meridional plane, i.e., one should not force $u_{z i} \equiv u_{z e}$ and $u_{r i} \equiv u_{r e}$. Imposing local current ambipolarity everywhere in the $2 \mathrm{D}$ model renders it mathematically incompatible[34]. Only in the full magnetization limit $\Omega_{i 0}=\infty$ local current ambipolarity can be kept, but this model conceals completely plasma detachment and is unsuitable for studying the far-region expansion of the plume[44].

Closure of the longitudinal electric currents must take place both downstream and upstream of the MN region considered here. Upstream, plasma resistivity within the plasma source should be effective for it. Downstream, electron demagnetization together with extended, weak resistivity should do the work.

\section{Propulsive performance}

After touring the essential plasma physics in the MN, the mechanisms of ion acceleration and thrust generation, and the downstream detachment of the jet, it is time to explore the propulsive performance of these devices. This section discusses two figures of merit of the $\mathrm{MN}$ : its thrust gain, and the divergence angle of the resulting plasma jet. 


\subsection{Thrust gain and plume efficiency functions}

Since the total thrust is the sum of the internal and magnetic thrust contributions, it is convenient to define a quantity that only depends on the performance of the MN itself, as independent as possible from the plasma source used. Such a quantity is the thrust ratio produced by the nozzle in the region between the throat and a normal section at $z$, or the thrust gain function,

$$
F(z) / F(0),
$$

where $F(z)$ can be computed using the conservation of momentum as the ion and electron momentum flux through the $z$ section,

$$
F(z)=2 \pi \int_{0}^{R_{V}(z)} n\left(m_{i} u_{z i}^{2}+T_{e 0}\right) r d r .
$$

Note that the ratio $F(z) / F(0)$ is akin (but not exactly the same) to a quantity commonly used in 'de Laval' nozzle literature, known as the thrust coefficient [45], i.e., the thrust of the nozzle divided by the throat area and the total pressure of the flow.

The ratio $F(z) / F(0)$ is depicted in Fig. 8(a) for several types of simulations, ranging in MN strength $\left(\hat{\Omega}_{i 0}\right)$, MN aperture (through the parameter $R_{L} / R$ in the case of a single current loop) and initial density profiles. The relative thrust gain is in all cases around 1.7-2. The main features of this thrust gain are as follows. First, most of the thrust gain is produced in the very near region of the MN, where the magnetic field is large, and plasma density and currents are large. This is in agreement with Fig. 6, where the maximum thrust force density is seen to occur at the beginning of the divergent MN. That is also the region where the ion Mach number increases rapidly from near-sonic to hypersonic: the ratio of electron thermal energy to ion kinetic energy is still large in this part of the MN, so ion acceleration occurs fast. The thrust gain increments become smaller further downstream.

Second, thrust gain at a given area expansion ratio [i.e., $\hat{R}_{V}^{2}$, with the jet radius $R_{V}(z)$ given in the horizontal axis of Fig. $8(a)$ ] decreases slightly with incrising ion magnetization degree, $\hat{\Omega}_{i 0}$. This is in line with the previous comments on the enhanced radial expansion and ion drag when $\hat{\Omega}_{i 0}$ is large. The effect, however, is minimal within the foreseen range of $\hat{\Omega}_{i 0}$ for propulsive applications $\left(\hat{\Omega}_{i 0}<100\right)$. The MN divergence rate has a larger influence on thrust: fast-diverging MNs (low $\hat{R}_{L}$ ) imprint a larger radial component to the plasma jet at early stages of the expansion, i.e. where most ion acceleration and thrust generation are taking place. This imposes a penalty on the axial component of ion acceleration, which therefore results in a lower thrust gain.

A clearer understanding of the influence of radial acceleration and the effect of $\hat{\Omega}_{i 0}$ can be obtained by introducing the plume efficency function, which is defined as the ratio of axial ion kinetic power to total ion kinetic power at $z[24]$,

$$
\eta_{\text {plume }}(z)=\frac{\int_{0}^{R_{V}(z)} r n u_{z i}^{3} d r}{\int_{0}^{R_{V}(z)} r n u_{i}^{2} u_{z i} d r} .
$$



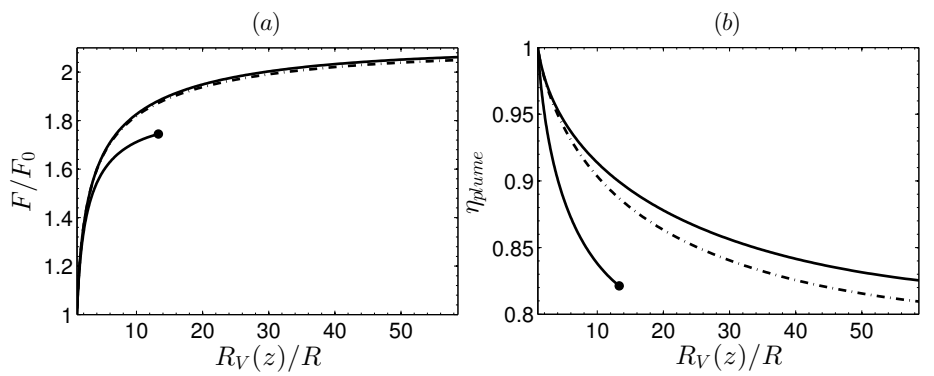

Figure 8: Variation along the nozzle of $(a)$ thrust gain $F(z) / F_{0}$ and plume efficiency $\eta_{\text {plume }}$, for several simulations with $R_{L} / R=3.5$ (shorter lines terminated with a circle) and $R_{L} / R=5.4$ (longer lines) and a non-uniform initial plasma profile based on the $J_{0}$ Bessel function. Both variables have been plotted against $R_{V}(z)$. Solid lines represent $\hat{\Omega}_{i 0}=0.1$, dash-dot lines represent $\hat{\Omega}_{i 0}=100$. Source: Adapted from Ref. [24]. (c) 2010 AIP. Adapted with permission.

This quantity is displayed for the same cases as before on Fig. 8(b) as a function of $R_{V}(z)$. It is apparent that a lower $\hat{\Omega}_{i 0}$ and a smaller aperture of the MN (higher $\hat{R}_{L}$ ) improves $\eta_{\text {plume }}$, and therefore the design goal for the MN is to have long, slowly-diverging magnetic fields with $\hat{\Omega}_{i 0}$ low, while still ensuring correct operation (i.e., electrons well magnetized).

Lastly, $F / F_{0}$ continues to grow logarithmically with $z$ and does not seem to approach an asymptotic value. The ambipolar electric potential behaves in the same way, and this is a setback common to isothermal plasma plume models[46, 41]. As it will be seen in Section 4.1, introducing some degree of electron cooling in the plasma beam leads to a finite electric potential drop and thrust gain.

A brief note on the specific impulse of the MN, $I_{s p}=F / \dot{m}$, is also due. $I_{s p}$ follows the same behavior as $F / F_{0}$; in fact, defining $I_{s p, 0}$ as the specific impulse at throat, it is clear that

$$
I_{s p}(z) / I_{s p, 0}=F(z) / F_{0},
$$

so its evolution along the nozzle is identical. As the bottom line of this discussion, observe that both $F_{0}$ and $I_{s p, 0}$ increase with the sonic velocity $c_{s}$, which in turn is proportional to $\sqrt{T_{e 0}}$. This illustrates how deeply the propulsive performance of the $\mathrm{MN}$ relies on having a high $T_{e}$ :

$$
I_{s p, 0} \propto c_{s} ; \quad F_{0} \propto R^{2} n_{0} c_{s}^{2}:
$$

It can be stated that the $\mathrm{MN}$ is an electromagnetic device from the viewpoint of the forces that create thrust, and an electrothermal device from the viewpoint of the conversion of energy. 


\subsection{Plume divergence}

While thrust production is the primary figure of merit of any propulsion system, there are other important aspects to consider when evaluating a particular device. The divergence angle of the plasma jet plays a major role in the integration of the thruster on the spacecraft, where a small angle is desirable to minimize the plasma interaction with the sensitive surfaces and equipment neighboring the thruster. A classical example of a detrimental plume interaction is with the solar arrays: the energetic plasma can erode, or deposit extraneous materials over the solar cell coverglass, reducing its efficiency and increasing the risk of discharges occurring between the different elements, potentially damaging them[47]. A lower plume divergence is also interesting from the propulsive viewpoint, as this is intimately linked with a high plume efficiency. In the same spirit, it is clear that maintaining a low divergence angle is linked to proper detachment. It is conventional to use the local divergence half-angle of the plasma tube containing $95 \%$ of the ion current, $\alpha_{d i v}$, to describe the divergence of the plasma jet. An alternative figure is the global divergence half-angle, computed as the half-angle of the smaller cone that envelopes the $95 \%$ of the plume.

The MN can in fact be regarded as a device that provides a means to control plume divergence, reducing it with respect to the unmagnetized expansion of a sonic plasma to vacuum. While all electric thrusters have some amount of backflowing plasma, the existence of a guiding magnetic field in the case of a MN could potentially reduce it[48]. The divergence follows the same trends as $\eta_{\text {plume }}$ : longer MNs and lower ion magnetization degrees produce lower divergence angles.

Figure 9 illustrates $\alpha_{d i v}$ as a function of the radius of the $95 \%$ tube. For this particular example, not optimized for low divergence, $\alpha_{\text {div }}$ goes from about 60-70 deg for $\hat{\Omega}_{i 0}=1$ (the global divergence angle is $53 \mathrm{deg}$ in this case) to up to more than $90 \mathrm{deg}$ for fully-magnetized ions (dashed line), when ion detachment does not occur at all and ion streamtubes coincide with magnetic streamtubes. However, it is clear that even very high ion magnetization values $\left(\hat{\Omega}_{i 0}=200\right)$ are still very far apart from the full ion magnetization limit curve. This agrees with the only mild differences observed in Fig. 7 between the two magnetization values. Comparing both figures, it is evident that as soon as ion detachment starts to grow significantly, the $\alpha_{d i v}$ line separates from the fully-magnetized limit line. This suggests $\alpha_{d i v}$ or the global divergence angle (a value easily measurable in experiments[26, 29, 31]), can be used as a good indicator for plasma detachment.

Lastly, these results show that the MN can operate at very high magnetic strengths, far larger than the ones envisioned for practical applications, before substantially affecting the propulsive performance of the device.

Interestingly, as with $F / F_{0}, \alpha_{\text {div }}$ does not exhibit a clean asymptotic behavior of downstream. This is related to the fact that the radial electric field that pushes ions outward decays logarithmically in the $\mathrm{MN}$ in an isothermal plasma. As discussed in Section 4.1, when electron cooling takes place in the plume the electric field decreases faster, and therefore the ion streamtubes approach a 


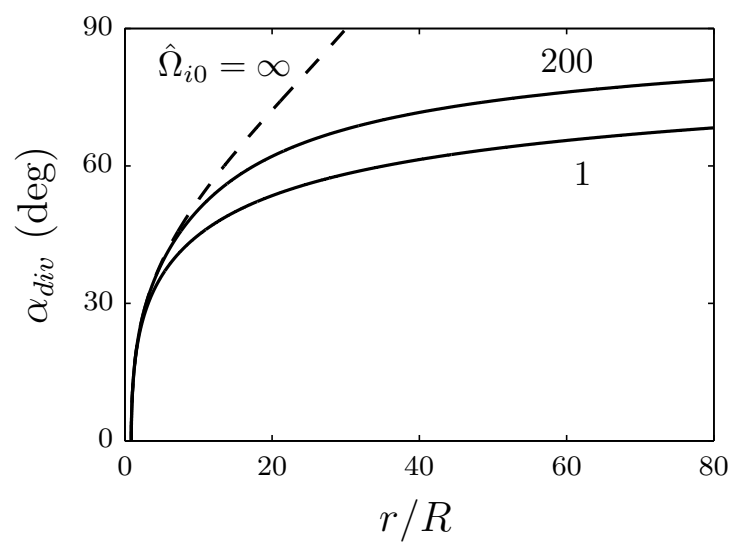

Figure 9: Evolution of the local divergence angle $\alpha_{d i v}$ in a non-optimized MN, as a function of the 95\%-mass flow tube radius, for the same simulations as in Fig. 7. The global, half-cone divergence angle computed from the data in the simulated region is 53 and $60 \mathrm{deg}$ for the $\hat{\Omega}_{i 0}=1$ and $\hat{\Omega}_{i 0}=200$ cases, respectively. The dashed line corresponds to the total ion-magnetization limit, $\hat{\Omega}_{i 0} \rightarrow \infty$ (see Ref. [44]). Source: Adapted from Ref. [35]. (C) 2014 IOP. Adapted with permission.

straight conic shape downstream.

\section{Advanced physics of magnetic nozzles}

The essential MN model described in Section 2.1 has served to illustrate the main aspects of the plasma expansion, thrust generation, and detachment. However, the model makes several simplifying assumptions that allow to neglect some physical phenomena in the MN. While most of these effects should have only a small effect in a well-designed MN, it is necessary to understand and assess their influence. Here, the basic model is gradually enriched by dropping one of its core assumptions at a time, exploring the significance of the associated phenomenon on the operation of the device.

\subsection{On the thermodynamics of electrons}

Up to this point, it has been assumed, for the sake of simplicity, that electrons are isothermal, under the hypothesis that the larger part of the population is confined by the ambipolar electric field, and only a small hot tail of the electron energy distribution function (EEDF) escapes downstream to maintain the globally-zero current condition. Actually, isothermal electrons are a justifiable assumption solely in the near-region of the MN, where most of the population is effectively confined by the electric field. Far downstream, the fraction of escaping electrons begins to dominate the EEDF, and the isothermal assumption 
cannot be justified any longer.

Indeed, isothermality is not fully consistent with an infinite expansion to vacuum. Obviously, as it can be inferred from Eq. (10), $\phi \rightarrow-\infty$ on each electron streamtube when $n \rightarrow 0$, which, albeit logarithmically slow, leads to an unphysical infinite potential drop and an infinite ion acceleration. This was the reason behind the unbounded growth of $F / F_{0}$ and $\alpha_{\text {div }}$ in Section 3.1.

As with any expanding species, electrons spend internal energy as they expand. To sustain the isothermal electron condition in the plasma domain, an electron kinetic heat flux $\boldsymbol{q}$ must flow from the plasma source, to replenish the electron thermal energy and keep it constant. Consequently, the total power that streams out of the plasma source is the sum of the initial ion kinetic power, the electron thermal power, and the electron heat flux; evaluating it at the throat:

$$
P_{0}=2 \pi \int_{0}^{R}\left(\frac{1}{2} m_{i} n u_{i}^{2} u_{z i}+\frac{5}{2} n T_{e} u_{z e}+q_{z}\right) r d r .
$$

Clearly, to maintain $T_{e}=T_{e 0}$ down to infinity would require an infinite supply of power at the plasma source in the form of an infinite electron heat flux[49]. This unphysical result, common to all isothermal plume models, indicates that there must exist some form of collisionless electron cooling that gradually decreases $T_{e}$.

In order to illustrate the influence of electron cooling on the expansion in a simple manner, the model of Section 2.1 is first extended to treat electrons phenomenologically as polytropic species[49], with

$$
\frac{T_{e}}{T_{e 0}}=\left(\frac{n}{n_{0}}\right)^{\gamma_{e}-1}
$$

where $\gamma_{e}$ is an effective electron cooling rate. Observe that $\gamma_{e}=1$ recovers the isothermal limit, whereas $\gamma_{e}=5 / 3$ would be the value for adiabatic electrons (i.e., no kinetic heat flux, $\boldsymbol{q}=\mathbf{0}$ ). Values between $\gamma_{e}=1.1$ and 1.3 are expected from several experimental observations[16, 27, 50] and kinetic simulations $[51,52,53]$. Nonetheless, there seems to be a case-by-case dependence of this parameter, as yet other experiments have reported higher $\gamma_{e}$, approaching a near-adiabatic behavior[54].

When this equation of state is employed, the electron equation can be written as:

$$
0=-h_{e}+e \nabla \phi-e u_{\theta e} B \mathbf{1}_{\perp}, \quad \text { where: } h_{e}=\frac{\gamma_{e}}{\gamma_{e}-1} T_{e}
$$

is the barotropy function for $\gamma_{e} \neq 1$, and as before, $h_{e}-e \phi=H_{e}(\psi)$.

The consequences of electron cooling are threefold. First, when $\gamma_{e} \neq 1$, in contrast with the isothermal limit there is an asymptote to the potential drop along the $\mathrm{MN}$, and therefore one for ion velocity too, with a clear dependence on $\gamma_{e}$ :

$$
e \phi \rightarrow-\frac{\gamma_{e}}{\gamma_{e}-1} T_{e 0} ; \quad u_{i} \rightarrow \frac{\gamma_{e}+1}{\gamma_{e}-1} c_{s 0} .
$$


(a)

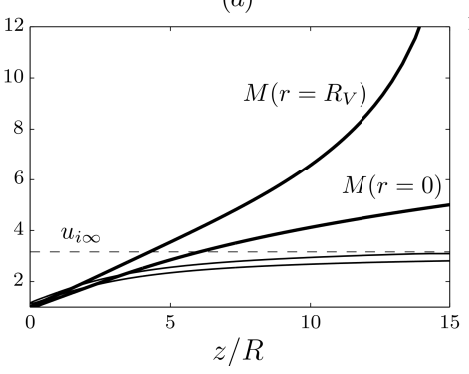

(b)

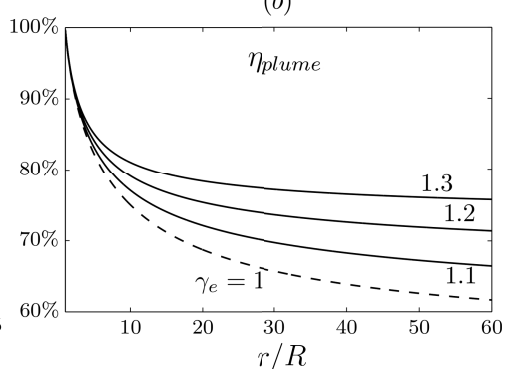

Figure 10: Ion Mach number (thick lines) and ion velocity (thin lines) at the plasma edge $\left(r=R_{V}\right)$ and the axis $(r=0)$ for $\gamma=1.3(a)$. $M$ is comparatively much higher than in the isothermal case. The asymptotic ion velocity $u_{i \infty}$ is shown as a dashed line. Plume efficiency for various values of $\gamma$, computed with the $95 \%$ ion plasma tube, and plotted against the radius of this tube $(b)$. Both figures use an initially uniform plasma density profile, and a MN with $R_{L} / R=3.5$. Source: Adapted from Ref. [49]. (C) 2015 IEEE. Adapted with permission.

Second, as depicted in Fig. 10(a), the decrease of $T_{e}$ in the polytropic electron expansion translates into a higher ion Mach number, i.e. the plasma pressure goes to zero faster. The main reason behind this is that in the polytropic model, the ion sonic velocity decreases with temperature, $c_{s}=\sqrt{\gamma_{e} T_{e} / m_{i}}$, while in the isothermal model it was constant. Since $\nabla \phi \sim T_{e} /(E R)$, the lower ambipolar electric field in the colder plasma means in turn that ion deflection is reduced, and therefore enhanced ion separation, leading to earlier plasma detachment, a lower plume divergence and a higher plume efficiency than in the isothermal case (Fig. 10(b)).

Third, while the ion Mach number increases faster in the polytropic plasma, the ion velocity gained at a given section of the $\mathrm{MN}$ is lower than in the isothermal case. This naturally means a lower (and actually upper-bounded) thrust gain $F / F_{0}$ in the $\mathrm{MN}$, as defined in Eq. (22).

This lower $F / F_{0}$ may lead to the wrong impression that a more isothermal electron behavior is desirable for the higher thrust gain in the MN. Note, however, that the electron kinetic heat flux $\boldsymbol{q}_{e}$, which accounts for the largest part of the plasma power when $\gamma_{e} \simeq 1$, is not contemplated at all in $F / F_{0}$. To compare the isothermal and polytropic nozzles fairly, a constant total power $P$ at the throat should be used, or, alternatively, refer instead to the thrust efficiency of the device, $\eta_{F}=F I_{s p} /(2 P)$.

Quantifying the detailed electron heat flux that leaves the plasma source is a complex task that cannot be afforded with the current model; in particular, this requires coupling it with an appropriate plasma source model. This prevents carrying out this analysis in terms of $\eta_{F}$ or $F / F_{0}$ at constant power. Moreover, kinetic electron models are required to obtain the actual evolution of $T_{e}$ and $\boldsymbol{q}_{e}$. 
Notwithstanding this, under some simplifying assumptions, simple estimates of the global electron heat flux at the MN throat can be made[49]. The general trend found from those estimations are as follows: the higher $\gamma_{e}$, the lower the kinetic electron heat flux at the throat, and the faster power conversion occurs (see Fig 10(a), where $u_{i}$ readily approaches its asymptote early in the expansion). This hints at a higher degree of power conversion into kinetic ion energy at a sooner stage. Ensuring early conversion is important, as the MN becomes weaker downstream and other effects may come into play that could hinder further energy conversion. This, together with the higher plume efficiency [Fig. 10(b)] and associated lower divergence, suggests that the efficiency should $\eta_{F}$ improve with electron cooling.

Finally, observe that, for the reasons stated above, the isothermal limit yields a conservative estimate of the plasma detachment and plume efficiency, making it a valuable tool for preliminary MN characterization and performance estimation (even if it leads to an unphysical infinite). The polytropic model can be used as a first approximation to study the effects of cooling, when an effective $\gamma_{e}$ is available from other sources of information (experiments or models).

A second important problem regarding the correct modeling of the EEDF is the assumption of isotropy. A magnetized, collisionless population may not, in principle, remain isotropic in the $\mathrm{MN}$, even if the plasma source conditions are collisional enough to provide an initially isotropic electron population at the throat. An anisotropic species exhibits magnetic mirror terms in its momentum equation, which do not appear explicitly in the isotropic case[55].

In order to understand the development of electron anisotropy, a full kinetic treatment of the EEDF is required. Navarro et al.[56] have recently addressed this problem with a fully-magnetized 1D-2 V model, showing that an initially isotropic expanding population has a first region of near-isothermal behavior, followed by a second region where cooling can be approximated by fitting a polytropic law (obtaining values of $\gamma_{e}$ in the range discussed in this Section). Finally, farther downstream, in a third region where the electrostatically-confined fraction of the population becomes negligible, the electron species begins to develop its anisotropicity, with the perpendicular temperature $T_{\perp e}$ going to zero while the parallel temperature $T_{\| e}$ tends to an asymptotic value. This is a result of the magnetic mirror effect acting on each individual electron that leaves the $\mathrm{MN}$, gradually converting its perpendicular energy into parallel energy. Moreover, the study of the EEDF of Ref. [56] reveals the complexity of the electron expansion, where electric potential barriers and the magnetic mirror effect combine to confine electrons depending on their energy and their magnetic moment, forming inaccessible regions of the phase space of the electron distribution function. The relevant parameter for cooling, anisotropy development, and barrier forming is seen to be the ion-to-electron mass ratio, $m_{i} / m_{e}$. Electrostatic barriers and anisotropization have also been studied by Arefiev et al. [57, 58] in the transient formation of the plasma jet.

The last point of discussion in this section concerns the assumption of electron Maxwellianicity in the expansion. Again, if collisionality inside the plasma source is sufficient to thermalize the electrons, they will leave the thruster with a 
Maxwellian EEDF at the throat. However, this could not be the case with some plasma thrusters, as at least some experiments with helicon plasma sources hint at the formation of a high-energy tail of electrons in coexistence with a colder, bulk population under certain operation modes[59, 60, 61]. Other nonMaxwellian EEDFs could conceivably arise in other devices. A strong nonMaxwellianicity can change substantially the profile of the electric field in the $\mathrm{MN}$, and give rise to electrostatic structures like double-layers[62, 63], potentially explaining some of the observed structures in certain experiments $[64,65$, 66]. To the extent that a hot-electron-tail EEDF can be modelled as a superposition of a cold and a hot Maxwellian populations, it is possible to explore the range of parameters in which these features appear, the shape and strength of the double-layer, and its effect on magnetic thrust and plume efficiency using a similar model to the one described here[62, 63]. Significantly, double-layers do not increase thrust or the efficiency of the device, which depend primarily on the total potential drop in the MN and the directionality of the ion acceleration. Double-layers seem however to increase the radial acceleration to ions due to their inherent 2D curvature[63], a detrimental effect on $\eta_{p l u m e}$ and $\alpha_{d i v}$.

\subsection{Influence of the type of internal energy in the plasma}

For most of the MN-based thrusters, it is a good assumption to consider $T_{e} \gg T_{i}$. Notwithstanding this, there are cases where ion internal energy plays an important role and cannot be neglected. A prominent example is the VASIMR thruster, where an additional ion cyclotron resonance heater, located downstream of the ionization helicon source, deposits energy directly onto the ions. The fraction of the thruster power dedicated to this heater can be large, leading to $T_{i} / T_{e}$ up to $\sim 10$.

Introducing ion temperature in the model is simple, needing only a minor modification to the ion momentum equation, Eq. (4):

$$
m_{i}\left(\boldsymbol{u}_{i} \cdot \nabla\right) \boldsymbol{u}_{i}=-\nabla h_{i}-e \nabla \phi+e \boldsymbol{u}_{i} \times \boldsymbol{B},
$$

where $h_{i}=\gamma_{i} T_{i} /\left(\gamma_{i}-1\right)$ for polytropic ions $\left(T_{i}=T_{i 0}\left(n / n_{0}\right)^{\gamma_{i}-1}\right)$, and $h_{i}=$ $T_{i 0} \ln \left(n / n_{0}\right)$ for isothermal ions. Moreover, the model with ion internal energy can be easily reduced to the cold ion model that has been used so far, by intro-

ducing an equivalent electric potential, $\tilde{\phi}=\phi+h_{i} / e$ and the plasma barotropy function $h=h_{e}+h_{i}$, so that $h_{i}$ no longer appears explicitly in the equations[49]. The appropriate sonic velocity is now given by $m_{i} c_{s}^{2}=\gamma_{i} T_{i}+\gamma_{e} T_{e}$.

The initial plasma conditions at the throat must still satisfy the equilibrium of forces in the radial direction. There are two basic possibilities for the plasma to achieve this: In the first case, the new ion pressure contribution is compensated by a diamagnetic ion drift, very much like the electron diamagnetic drift confines the electron pressure. In this case, the electric potential and the electron equilibrium remain basically unaltered at the throat. In the second case, ion pressure is confined electrostatically. The new non-zero electric field at the throat now demands a higher electron azimuthal velocity $u_{\theta e}$; in effect, electron 


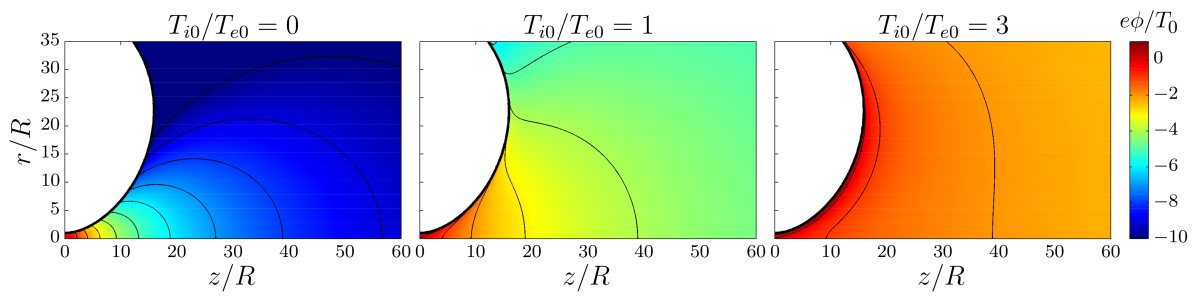

Figure 11: Electric potential $\phi$ for three values of $T_{i 0} / T_{e 0}$. The simulations use an initially nonuniform plasma jet and the $R_{L} / R=3.5 \mathrm{MN}$. To facilitate fair comparison, the electric potential has been normalized with the total temperature $T_{0}=T_{i 0}+T_{e 0}$. Solid lines represent isopotential surfaces in the plasma, spaced in increments of $e \phi / T_{0}=1$. Source: Adapted from Ref. [49]. (C) 2015 IEEE. Adapted with permission.

currents are then in charge of confining both the electron and ion pressure. At any rate, a intermediate case between the two extremes will occur, and the total azimuthal current must balance the total plasma pressure (ion plus electron) at the throat, $j_{\theta}=j_{\theta i}+j_{\theta e} \sim n\left(T_{i}+T_{e}\right) / B$.

Both ion and electron internal energies can be used to power the expansion and the generation of thrust. In the case of electron energy, this is transferred to ions thanks to the ambipolar electric field as described already. The magnetic mirror effect helps to transform the perpendicular electron energy into parallel electron energy, so it is not lost into pointlessly accelerating the plasma radially. In the case of ion energy, the ambipolar electric field is not involved in the acceleration: the perpendicular ion energy is transformed into parallel energy by the magnetic mirror effect on ions, when ions are magnetized, or by electrostatic reflections on the beam edge otherwise. The latter mechanism resembles the classical gasdynamic acceleration in a de Laval nozzle with solid walls, where particles bounce off them.

Nevertheless, magnetic thrust must still be generated by the interaction of the applied magnetic field with the total azimuthal current in the plasma, $j_{\theta}=j_{\theta i}+j_{\theta e}$. It is the total current, and therefore the total plsama pressure, that defines the thrust gain in the MN. In the case that the ion azimuthal velocity were $u_{\theta i}<0$ initially to confine the ion pressure, the ion current would be diamagnetic (at least initially) and would provide a positive thrust contribution, contrary to the $u_{\theta i}(0, r)=0$ case, where $u_{\theta i} \geq 0$ is always paramagnetic.

To illustrate the effects of ion internal energy in a simple case, Fig. 11 displays three simulations with various values of the ratio $T_{i 0} / T_{e 0}$, assuming both species are isothermal and that the initial radial confinement is afforded by the electrons only (i.e., $u_{\theta i}=0$ and $\phi=0$ at the throat).

In agreement with the discussion so far, $e \nabla \phi \propto T_{e 0} / R$, and the first observed effect in Fig. 11 is that the intensity of the ambipolar electric field normalized with the total temperature $T_{0}=T_{i 0}+T_{e 0}$ is lower the higher $T_{i 0} / T_{e 0}$ is, revealing a higher role of ion gasdynamic acceleration. Secondly, the existence of ion 
energy causes the initial radial electric field to change from ion-expanding to ionconfining. This can be inferred from the shape and curvature of the isopotential lines in Fig. 11. Downstream, where magnetic/electric tube turning is large and ion inertia dominates in the ion equation, the electric field becomes ionexpanding in all the considered cases, albeit this occurs farther downstream the higher $T_{i 0} / T_{e 0}$.

In summary, the presence of ion internal energy introduces new mechanisms for ion acceleration, and can dramatically change the electric potential profile within the plasma. Nonetheless, the generation of thrust still depends on the azimuthal electric currents, which respond to the total internal energy in the plasma regardless of which species carries it.

\subsection{Plasma-induced magnetic field}

When the plasma is sufficiently dense and hot, the very same electric currents that are responsible for thrust generation and radial confinement induce a magnetic field that can modify the shape and strength of the MN. In essence, as stated in Section 2.1, the total magnetic field $\boldsymbol{B}$ is the sum of the applied and the plasma-induced one, $\boldsymbol{B}=\boldsymbol{B}_{a}+\boldsymbol{B}_{p}$. So far, the plasma-induced field has been neglected assuming a low-density plasma, but it can be readily included in the model by adding Ampere's equation:

$$
\nabla \times \boldsymbol{B}_{p}=\mu_{0} \boldsymbol{j} .
$$

While all components of $\boldsymbol{j}$ create an induced field, it is the $j_{\theta}$ component that modifies the meridional magnetic field, thus altering the shape of the MN. Moreover, in most cases of interest, this current is the largest component of $\boldsymbol{j}$. Thus, keeping only the azimuthal projection of Eq. (31), one may write it in terms of $\psi_{p}$, the induced magnetic field streamfunction, as

$$
\frac{1}{r} \frac{\partial^{2} \psi_{p}}{\partial z^{2}}+\frac{\partial}{\partial r}\left(\frac{1}{r} \frac{\partial \psi_{p}}{\partial r}\right)=\mu_{0} j_{\theta},
$$

which exhibits explicitly the elliptic nature of the problem of calculating $\psi_{p}$.

Recalling that in a cold-ion plasma $j_{\theta e} \sim n_{0} T_{e} /\left(B_{a} R\right)$, a quick estimate in the equation above shows that the ratio $B_{p} / B_{a}$ scales as

$$
\frac{B_{p}}{B_{a}} \sim \mu_{0} n T_{e} / B_{a}^{2}=\beta,
$$

which is the well-known plasma-beta parameter. Its value at the center of the throat section, $\beta_{0}$, can be used to characterize the influence of the plasmainduced magnetic field[67]. A possible scheme to compute the self-consistent magnetic field is to calculate $\boldsymbol{B}_{p}$ iteratively, obtaining first a solution for $j_{\theta}$ and then calculating the induced field. The integration of the plasma flow and the calculation of $\boldsymbol{B}_{p}$ can be repeated with the new total field until convergence is achieved[67]. 

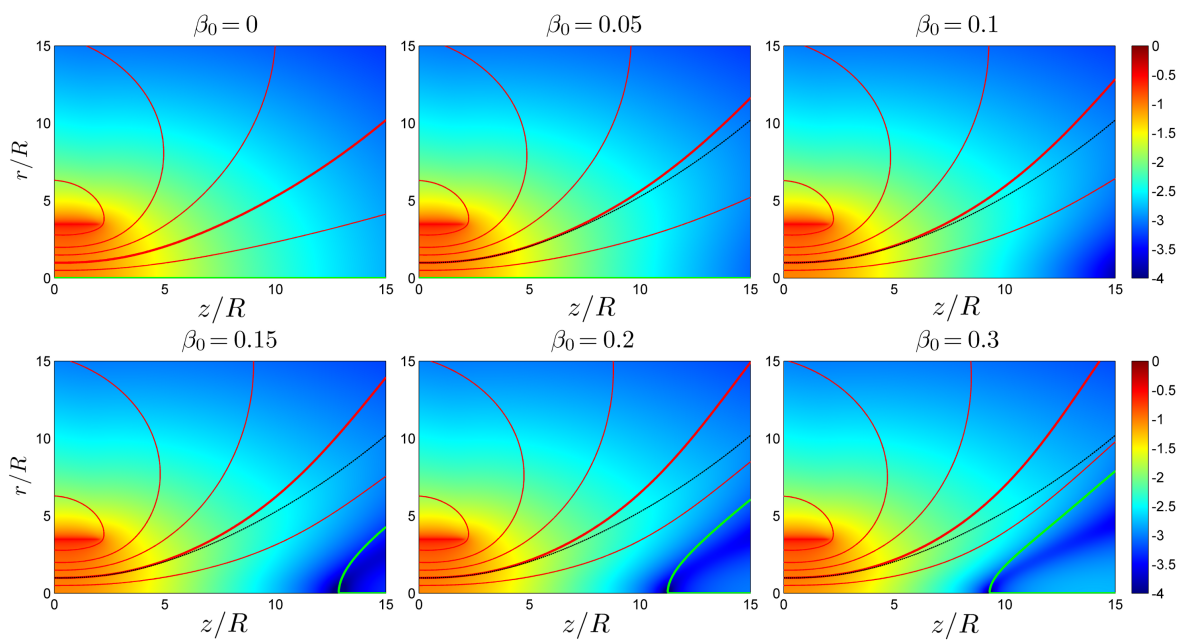

Figure 12: Total magnetic field $\boldsymbol{B}=\boldsymbol{B}_{a}+\boldsymbol{B}_{p}$ calculated self-consistently for different values of $\beta_{0}$. An isothermal plasma with an initial profile based on the $J_{0}$ Bessel function was used. In this case, the magnetic nozzle is generated by a series of point coils that simulate a solenoid analogous as the one used in several helicon plasma thrusters. The color map represents $\log _{10} B$. Red lines show magnetic streamtubes, the thicker one being the plasma-vacuum edge in the simulations (the line that passes by $z=0, r=R$ ). The black dotted line shows the position of this plasma edge in the $\beta_{0}=0$ simulation for comparison. The green line, when present, displays the magnetic separatrix, for which $B=0$ at the axis. Since the model cannot predict the value of $u_{\theta e}$ downstream from the separatrix, $u_{\theta e}=0$ has been used in that region.

The first observation regarding $\boldsymbol{B}_{p}$ is that it opposes the applied magnetic field. This is not surprising, since the azimuthal plasma currents $j_{\theta}$ are naturally diamagnetic in the MN. This was also a necessary condition for positive thrust generation. As a result, the induced field weakens the magnetic strength in the $\mathrm{MN}$ and makes the magnetic lines more rapidly divergent; in other words, the MN becomes shorter and wider, with the turning point closer to the throat region. Figure 12 illustrates the effect of increasing the $\beta_{0}$ parameter, showing that MN opening increases with $\beta_{0}$.

Second, in this isothermal case, the induced field gains relevance downstream. The reason is that $n T_{e}$ decreases slower than $B_{a}^{2}$. That is, the local $\beta$ increases with the expansion. Note that this trend could change if rapid electron cooling takes place in the MN. As some of the simulations show, the induced magnetic field may become strong enough to fully cancel the applied one at the axis, forming a separatrix surface between two distinct regions of the MN: the applied-field-dominated one, and a reversed-field region downstream. The existence of a point where $B=0$ on the axis of the MN has been recently 
observed experimentally by Roberson et al.[68], and configurations where the core of the plasma has completely expelled the applied field where proposed by Gerwin[69].

Irrespective of the existence of a separatrix, the gradual expulsion of the magnetic field occurs mostly in the core of the MN, where plasma density is largest. However, the magnetic field strength in the periphery remains in the same order of magnitude in all cases considered here. This is important in a double sense: first, the demagnetization of the core facilitates detachment of the main part of the plasma jet. Second, the high magnetic field at the periphery helps to continue to confine the radial expansion of the plasma, minimizing its cross-field diffusion.

Notwithstanding this, simulations reveal a minor decrease in plume efficiency $\eta_{\text {plume }}$ with increasing $\beta_{0}$. Therefore, in a properly designed $\mathrm{MN}$ it is desirable to keep a low $\beta_{0}$, so that induced field effects do not disturb the geometry of the $\mathrm{MN}$ too early in the expansion (i.e. before a high ion Mach number is achieved), which would lead to an unwanted increase of plume divergence. This sets an upper boundary to plasma density for a given $B_{a 0}$ and $T_{e 0}$.

It is worth pointing out that these trends may change if a non-propulsive $\mathrm{MN}$ is considered, into which a hypersonic (or cold) plasma is injected, or when the ion magnetization strength is excessively high downstream. In this case, the plasma electric currents can be dominantly paramagnetic; this type of currents lead to an induced magnetic field that reinforces the applied one, closing and stretching it downstream instead of opening it (see, e.g., Arefiev et al.[33] or Winglee et al.[70]). Recall, however, that such paramagnetic currents produce negative thrust (i.e., drag). Finally, note also that while the force of the induced magnetic field on the MN field generator causes the magnetic thrust, the selfforce that the induced field $\boldsymbol{B}_{p}$ exerts on the plasma itself is purely internal (i.e., both action and reaction are in the plasma), and therefore this self-induced force cannot directly provide thrust.

\subsection{Collisional processes in the expansion}

A higher electron collisional frequency $\nu_{e}$ will reduce the Hall effect parameter, $\chi_{H}=\Omega_{e} / \nu_{e}$, calling into question one of the key hypotheses of the electron model used here. Electron collisions provide a means for cross-field diffusion, which so far has been neglected by assuming electrons follow perfectly the magnetic streamtubes (i.e., $u_{\perp e} \equiv 0$ ).

The dominant effect of electron collisions appears in the azimuthal electron momentum equation, which was dropped before in favor of the aforementioned assumption. If $u_{\perp e} \neq 0$ is now allowed, using the simplest model for collisions and assuming $\boldsymbol{B}$ points downstream as before, this equation reads:

$$
0=e u_{\perp e} B-m_{e} \nu_{e} u_{\theta e},
$$

or, in other words,

$$
u_{\perp e}=u_{\theta e} / \chi_{H},
$$


(b)

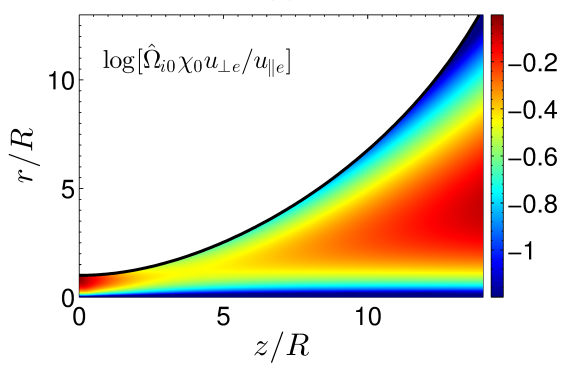

Figure 13: Diffusion caused by ion-electron collisions for a MN with $R_{L} / R=3.5$ and an initial plasma density profile based on the $J_{0}$ Bessel function. The color map shows $u_{\perp e} / u_{\| e}$ computed as a correction of the unperturbed solution $\left(\chi_{H}^{-1}=0\right)$. The plot has been normalized with $\chi_{0}$, the value of the Hall parameter at the center of the throat; $\hat{\Omega}_{i 0}$ has been included in the expression too, to take into account that $u_{\perp e}$ (just like $u_{\theta e}$ ) scales with $\hat{\Omega}_{i 0}^{-1}$. Therefore, except for the small variations in the zeroth-order density map with $\hat{\Omega}_{i 0}$, this graph applies to all values of $\hat{\Omega}_{i 0}$.

so that electron and magnetic streamtubes no longer coincide. Observe that in the collisionless limit $\left(\chi_{H} \gg 1\right) u_{\perp e}=0$ is consistently recovered. Collisions in the $z$ and $r$ electron momentum equations depend on $\left(u_{z e}-u_{z i}\right)$ and $\left(u_{r e}-u_{r i}\right)$ respectively, and play a secondary role since azimuthal currents are typically the largest in the plasma. Therefore, the focus of the discussion in this section are collisional effects in the azimuthal equation, Eq. (35).

Since $u_{\theta e}>0$ when $B_{z}(0,0)>0$ (see Section 2.3), it is clear without any further ado that $u_{\perp e}>0$, meaning that collisions result in a more divergent plasma jet due to diffusion.

Confirmation of this result and a preliminary estimate of the magnitude of $u_{\perp e}$ can be obtained assuming $\chi_{H}^{-1} \ll 1$ and computing the cross-field diffusion as a first-order correction using the unperturbed solution of the model[25]. Fig. 13 maps the value of $u_{\perp e} / u_{\| e}$ in an illustrative case, where $\nu_{e}$ is modeled as the ion-electron Coulomb collision frequency $\nu_{e i}$ (see, e.g. Ref. [71]). This plot shows that maximum diffusion is reached somewhere between the MN axis and the plasma periphery. This is due to the fact that $\chi_{H}^{-1}$ is higher at the axis, where density is larger, and decreases radially, while $u_{\theta e}$ is maximum near the periphery and zero at the axis; therefore the product of the two is nonmonotonic. Diffusion increases slowly downstream due to the growth of $u_{\theta e}$ in this direction.

In the foreseen application cases, $\chi_{H 0}$ (the Hall parameter at the center of the throat) ranges between $10^{2}$ and $10^{4}$ (Ref. [72]). Therefore, diffusion due to classical ion-electron collisions is expected to have a small influence in the MN expansion. 
(a)

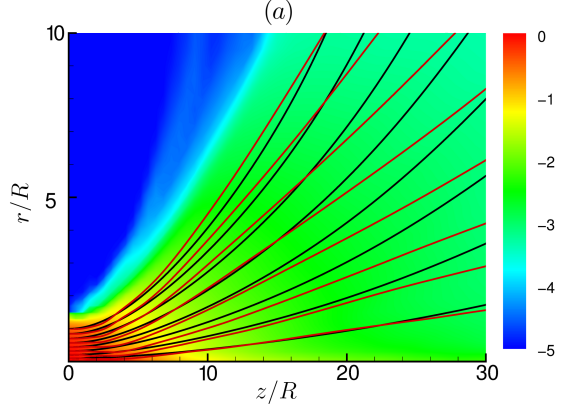

(b)

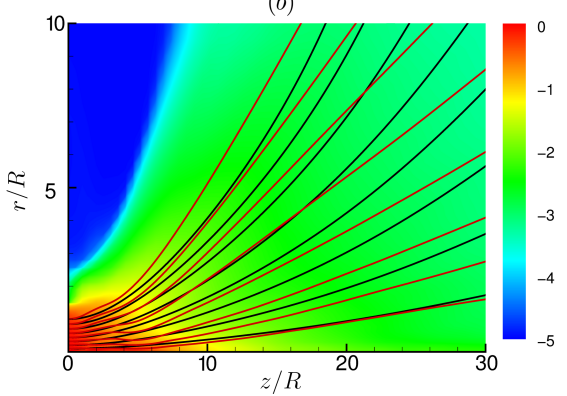

Figure 14: Plasma expansion in the MN as modeled with the hybrid PIC/fluid code of Ref. [48], for the $R_{L} / R=4.5$ nozzle with an initial density profile based on the $J_{0}$ Bessel function. Graph $(a)$ has $\chi_{H}=500$, graph $(b)$ has $\chi_{H}=100$. The colormap shows the plasma density in logarithmic scale, $\log _{10}\left(n / n_{0}\right)$. Red lines are ion streamtubes, black lines are the initially corresponding magnetic streambtubes (the outermost black line would be the plasma edge in the collisionless limit).

A more detailed simulation is shown in Fig. 14, based on a particle-in-cell code for ions and the electron fluid equations with collisions described above[48]. This hybrid approach allows us to transition from the fully-magnetized electron model to the case where collisions induce a considerable amount of lateral diffusion, and calculate the plasma flow self-consistently. Simulation $(a)$ has a higher magnetic field than simulation $(b)$.

Visibly, decreasing $B$ results in a wider, more divergent plasma jet. In fact, the reasons for this are two-fold: first, the lower $B$ results in a lower $\chi_{H}$, thereby increasing the ratio $u_{\perp e} / u_{\theta e}$. Second, a lower $B$ also means higher electron azimuthal currents (since $j_{\theta} B$ must remain the same at the throat to confine the plasma pressure); the higher $u_{\theta e}$ is, the larger the effect of the resistive term in Eq. (35), and thus the higher $u_{\perp e}$.

Figure 14 illustrates as well the growth in ion streamtube divergence near the periphery of the plasma when $\chi_{H}$ decreases. In this region, the outward electrostatic pull on ions is increased due to the enhanced electron diffusion. Most of this phenomenon occurs in the near region, where ion velocity is still low. Once the ions have gained enough velocity, however, their streamtubes eventually straighten out and become nearly conic, as was already seen in Section 2.4.

The hybrid model also allows to identify the minimum reasonable magnetic field strength to maintain good electron confinement in a given case, so that the $\mathrm{MN}$ confers its shape to the plasma jet and operates as expected: on the one hand, at very low magnetic strength the $\mathrm{MN}$ is too weak to channel and guide the plasma, and the expansion covers all space and has a very wide divergence angle. The magnetic thrust obtained is low. On the other hand, a very high magnetic strength leads to an undesirable increase of plume divergence due 
to higher ion attachment. Besides, a higher magnetic field requires a more expensive field generator, in terms of weight and power. Therefore, there exists an optimum range of magnetic field intensity for propulsive applications. This is actually the recovered behavior in the experiments of Ref. [50].

Lastly, observe that in agreement with the discussion, collisionality always leads to divergent detachement in a hot plasma MN, i.e., it causes the jet to diverge faster than the magnetic field. In consequence, it is not a useful detachment mechanism for propulsion (cf. with Ref. [32]), and therefore it should be avoided $[25]$.

\subsection{Influence of electron inertia}

The last assumption to be removed, at least partially, is that of negligible electron inertia. This simplification had multiple benefits for the tractability and integrability of the model; namely, the electron momentum equation becomes algebraic when the advective term is dropped, and the electron Larmor radius goes to zero, so that the position of individual electrons coincide with their gyrocenters.

Just like in the case of collisions, it is worth to analyze separately meridional and azimuthal electron inertia, for they become relevant at a different scale: on the one hand, in the meridional electron equations [Eq. (10)] the pressure term sets the leading order, and the electric and magnetic terms balance it. Therefore, longitudinal inertia plays a second-order effect, becoming relevant only when it is comparable to them. On the other hand, the azimuthal electron equation does not include a pressure term, and therefore the inertial one, irrespective of its magnitude, can set the leading order[34]:

$$
\frac{u_{r e}}{r} \frac{\partial\left(r u_{\theta e}\right)}{\partial r}+u_{z e} \frac{\partial u_{\theta e}}{\partial z}=\frac{e B}{m_{e}} u_{\perp e} .
$$

Hence, one can study the dominant effect of inertia by keeping only the azimuthal terms, and neglecting the meridional ones. Note that Eq. (36) can be integrated once into an expression analogous to Eq. (17), which states the conservation of the electron canonical angular momentum about the MN axis.

As with the analysis of collisions in Section 4.4, Eq. (36) yields $u_{\perp e} \neq 0$, so electron streamtubes do not coincide with magnetic tubes either. Expectedly, the limit $m_{e} \rightarrow 0$ reduces this equation to the condition used in the essential model, $u_{\perp e}=0$, too.

In this case, however, the addition of Eq. (36) to the model can be done self-consistently and it does not necessarily complicate its integration. In fact, after some algebra it can be shown that[34]: $(i) u_{\perp e}>0$ in the MN, leading to divergent detachment; and $(i i) u_{\theta e}$ and $u_{\perp e}$ can be computed a priori from the given magnetic field and initial plasma conditions, and therefore the electron streamlines are known before starting the integration of the ion flow. In fact, the perpendicular velocity scales as

$$
u_{\perp e} \propto \frac{\ell_{e}}{R} \frac{u_{r e} u_{\theta e}}{\sqrt{T_{e} / m_{e}}}
$$




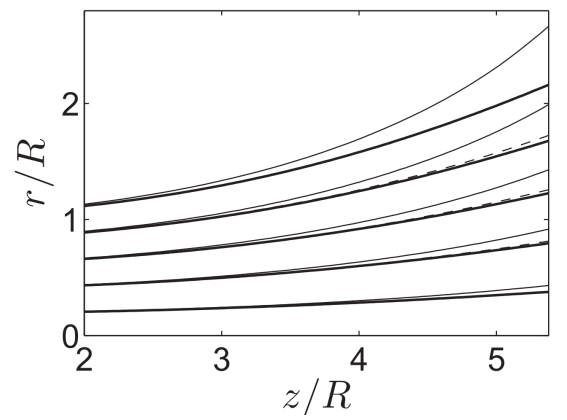

Figure 15: Initially-corresponding streamtubes of magnetic field (solid, thick), electrons (solid, thin), and ions (dashed) when azimuthal electron inertia effects are included in the model. An isothermal plasma with a Gaussian initial plasma density profile is used. In this case, the magnetic nozzle is generated by a solenoid and $\hat{\Omega}_{i 0}=0.4$, with $m_{e} / m_{i}=4.18 \cdot 10^{-6}$ (corresponding to Xe ions). Source: Adapted from Ref. [34]. (c) 2012 AIP. Adapted with permission.

in the bulk of the plasma, revealing the main dependency on the electron Larmor radius. Moreover, inertial effects are more marked where $u_{\theta e}$ is large.

These points are supported by the simulation of Fig. 15, where electron tubes are seen to separate outward from the magnetic field due to the azimuthal electron inertia. The separation increases toward the plasma edge, where $u_{\theta e}$ is larger. In this example, the increased electron separation pulls the ions radially, causing them to diverge more than the magnetic lines in the near region. Nonetheless, ion streamtubes still become near-conic farther downstream, so they eventually separate inward from the magnetic field in the bulk of the plasma just as in Fig. 7 .

Therefore, it can be concluded that azimuthal electron inertia does not facilitate inward plasma detachment (cf. with Ref. [22]), and just as collisional effects, it is desirable to keep these effects to a minimum (by increasing the applied field, for instance).

As a final comment, inertial effects can be regarded as a part of the broader, more complete 'finite Larmor radius effects' (FLRE), which scale with the electron Larmor radius $\ell_{e}$. These effects are due to the coherent gyration of all electrons about the magnetic lines, and have a dependency on the geometry of $\boldsymbol{B}$. One zeroth-order such effect is the appearance of the diamagnetic azimuthal current given by $-\left(\partial p_{e} / \partial r\right) / B$ at the throat. Azimuthal inertia is a first-order one, yet it is not the only one: together with this term, other contributions appear in the electron momentum equation, such as the gyroviscous force[73, 74], which has its origin in a non-isotropic, non-diagonal pressure tensor $\mathcal{P}_{e}$. Such terms appear even in the case of a isotropic, Maxwellian distribution of gyrocenters [73], and result naturally from the integration of the kinetic equations of the gyrating species. It is known that the gyroviscous force can cancel at least partially the effect of azimuthal inertia in the MN discussed here[34]. 
Modeling the complex gyroviscous terms and validating the conclusions of this section is currently an open topic of research. Moreover, FLRE will dominate far downstream, and be a substantial mechanism in electron demagnetization, a feature whose study will likely require full kinetic models.

\section{Conclusions}

This review of magnetic nozzle plasma physics has hopefully conveyed to the reader the key features and advantages of MNs for electric propulsion in space missions. MNs constitute an advanced plasma acceleration technology, with many attractive characteristics and the potential to perform efficiently, and are currently regarded as a promising alternative to other acceleration techniques. The many next-generation thrusters that are betting on MNs (HPT, VASIMR, AF-MPDT, ECRT, etc.) serve as a sample of the growing interest in these devices.

A 2D, 2-fluid model has been used to explore the basic physics and assessed the propulsive performance of the MN. While the main ion acceleration mechanism (and energy conversion mechanism) is the ambipolar electric field, magnetic thrust generation stands out from the existence of diamagnetic currents in the plasma and their interaction with the applied field; these currents are proportional to the pressure of the plasma (electrons plus ions) upstream.

With regards to plasma detachment, which has been one of the main concerns about MNs in the past decade, it has been shown to take place naturally thanks to the separation of the hypersonic ions from the field, when the electric and magnetic forces in the plasma become insufficient to deflect the ions radially along the magnetic tubes. Efficient detachment occurs even when their initial magnetization degree is far larger than the foreseen application ranges.

Lastly, by gradually extending the basic model, several additional aspects of the physics of MNs have been studied to understand their influence on the propulsive worthiness of the device. Electron cooling must take place in the expansion, and modeling it as an effective polytropic law revealed an overall improvement of MN performance with respect to the isothermal limit used in the basic model. Ion energy can modify strongly the ambipolar electric field in the plasma, and gives rise to the gas-dynamic acceleration mechanism. Induced field, collisions and electron inertia effects play a detrimental role, increasing the plume divergence and decreasing the plume efficiency.

\subsection{Open areas of research}

This last section gathers some of the main open loops in MN modeling, and comments on the difficulties that need to be overcome in order to advance in the mastering of MN physics for plasma propulsion.

The main limitations of the basic model used here lie on its central assumptions: fully quasineutral, collisionless, fully magnetized electrons, etcetera. Many of the kinetic subtleties of the expansion are concealed by the two-fluid 
nature of the model. Neutral atoms or a background plasma or magnetic field have been disregarded. Breaking free of these assumptions, and gradually incorporating kinetic effects in the model, is one of the next steps toward a better understanding of MNs.

In particular, one of the big open questions concerns the fate of the electrons far downstream, once that the magnetic field becomes weak enough for electron demagnetization to take place. The transition from the fully-magnetized electrons plasma into an unmagnetized electrons plasma jet is not a regular one, unless inertial or collisional effects are included; expectedly, FLRE will become a central aspect and will have to be consistently included up to first order. Being able to model electron demagnetization would allow to analyze the electron behavior in regions where the induced magnetic field dominates the applied one, too.

The low plasma density and the large Debye length at the periphery of the plasma (and far downstream) could bring up non-neutral effects locally if the Debye length $\lambda_{D}$ becomes larger than the macroscopic scale length, with unforeseen consequences on the expansion. Including Poisson's equation into the model is required to analyze this phenomenon.

Finally, the interaction of the MN plasma jet with the ambient plasma and/or magnetic fields remains to be addressed. Turbulence and two-stream instabilities could conceivably be present where the jet and ambient densities become comparable, and the ambient plasma will dictate the electric field when its density begins to dominate.

A full kinetic description of the plasma, in particular of the electrons, would allow to study the anisotropization and cooling of the species consistently. Preliminary results in this field using a 1D model are promising[56], and a 2D model that relaxes the full magnetization ccondition should clarify several open questions.

The joint, consistent treatment of the whole ensemble, plasma source plus $\mathrm{MN}$, to study the performance and operation of a complete thruster, is also under way in the case of the HPT[75]. That joint study can also clarify the closure of longitudinal electric currents inside the resistive plasma source.

Experiment-wise, while the main operation principles and plasma detachment have already been demonstrated, a complete parametric investigation of the expansion remains to be accomplished. Part of the difficulty lies on making the results as independent as possible from the vacuum chamber, test equipment and plasma source used. Eventually, space testing will become necessary to fully characterize the expansion and qualify this technology for flight.

Future developments and improvements of current MNs can also take place in the coming years. For instance, by using a 3D magnetic field instead of a $2 \mathrm{D}$, axisymmetric one, it is possible to guide the plasma laterally and deflect the jet to gain thrust vector control (TVC) capabilities[76]. While all existing thrusters need to be mounted on heavy gimbaled platforms to enable TVC, the use of a steerable MN could eliminate the need of such devices.

Another possible development stems out of the MN integration into the spacecraft, as the magnetic generator required to create the MN could be 
reused for other purposes. Examples of these are the generation of artificial magnetospheres[77] to protect the spacecraft from the harsh radiation environment of space, or its usage as a large magneto-torquer to reorient it.

Finally, MN plasma jets may also have an unexpected advantage over unmagnetized jets for certain applications. For example, the applied magnetic field may provide a means to shield the plasma expansion from the influence of external magnetic fields, such as the geomagnetic field, which may otherwise deform it[41]. While this may seem an innocent statement, it could have a central effect on applications that require targeting with precision an object in space with a plasma jet, such as the Ion Beam Shepherd (IBS) concept to deorbit space debris[78, 79].

\section{Acknowledgments}

The authors would like to thank the personnel at ESA EPS laboratory, SENER Ingeniería y Sistemas, and Jaume Navarro for the picture in Fig. 1, and Jaume Navarro for his help preparing Fig. 14. This work was supported by Spanish R\&D National Plan (grant number ESP2013-41052-P).

\section{References}

[1] R. Jahn, Physics of Electric Propulsion. Dover, 2006.

[2] E. Ahedo, "Plasmas for space propulsion," Plasma Physics and Controlled Fusion, vol. 53, no. 12, p. 124037, 2011.

[3] C. Charles, R. Boswell, and M. Lieberman, "Xenon ion beam characterization in a helicon double layer thruster," Applied Physics Letters, vol. 89, no. 26, p. 261503, 2006.

[4] O. Batishchev, "Minihelicon plasma thruster," IEEE Transaction on Plasma Science, vol. 37, no. 8, pp. 1563-1571, 2009.

[5] D. Pavarin, F. Ferri, M. Manente, D. Curreli, Y. Guclu, D. Melazzi, D. Rondini, S. Suman, J. Carlsson, C. Bramanti, E. Ahedo, V. Lancellotti, K. Katsonis, and G. Markelov, "Design of 50W helicon plasma thruster," in 31th International Electric Propulsion Conference, IEPC 2009-205, 2009.

[6] J. C. Sercel, "Electron-cyclotron-resonance (ECR) plasma acceleration," in AIAA 19th Fluid Dynamics, Plasma Dynamics and Lasers Conference, 1987.

[7] G. Krülle, M. Auweter-Kurtz, and A. Sasoh, "Technology and application aspects of applied field magnetoplasmadynamic propulsion," J. Propulsion and Power, vol. 14, no. 5, pp. 754-763, 1998. 
[8] V. Tikhonov, S. Semenikhin, J. Brophy, and J. Polk, "Performance of $130 \mathrm{~kW}$ MPD thruster with an external magnetic field and $\mathrm{Li}$ as a propellant," in Proceedings of the 25 th International Electric Propulsion Conference, pp. 728-733, 1997.

[9] H. B. Tang, J. Cheng, C. Liu, and T. M. York, "Study of applied magnetic field magnetoplasmadynamic thrusters with particle-in-cell and monte carlo collision. II. Investigation of acceleration mechanisms," Physics of Plasmas, vol. 19, no. 7, p. 073108, 2012.

[10] F. Diaz, J. Squire, R. Bengtson, B. Breizman, F. Baity, and M. Carter, "The physics and engineering of the VASIMR engine," in 36th AIAA/ASME/SAE/ASEE Joint Propulsion Conference \& Exhibit, AIAA 2000-3756, 2000.

[11] R. Hoyt, J. Scheuer, K. Schoenberg, R. Gerwin, R. Moses, and I. Henins, "Magnetic nozzle design for coaxial plasma accelerators," IEEE Transactions On Plasma Science, vol. 23, no. 3, pp. 481-494, 1995.

[12] K. Schoenberg, R. Gerwin, R. Moses, J. Scheuer, and H. Wagner, "Magnetohydrodynamic flow physics of magnetically nozzled plasma accelerators with applications to advanced manufacturing," Physics of Plasmas, vol. 5, no. 5, pp. 2090-2104, 1998.

[13] S. Andersen, V. Jensen, P. Nielsen, and N. D'Angelo, "Continuous supersonic plasma wind tunnel," Phys. Fluids, vol. 12, no. 3, pp. 557-560, 1969.

[14] K. Kuriki and O. Okada, "Experimental study of a plasma flow in a magnetic nozzle," Physics of Fluids, vol. 13, no. 9, p. 2262, 1970.

[15] T. M. York, B. A. Jacoby, and P. Mikellides, "Plasma flow processes within magnetic nozzle configurations," Journal of Propulsion and Power, vol. 8, no. 5, pp. 1023-1030, 1992.

[16] M. Inutake, A. Ando, K. Hattori, H. Tobari, and T. Yagai, "Characteristics of a supersonic plasma flow in a magnetic nozzle," J. Plasma Fusion Res., vol. 78, no. 12, pp. 1352-1360, 2002.

[17] H. Kosmahl, "three-dimensional plasma acceleration through axisymmetric diverging magnetic fields based on dipole moment approximation," tech. rep., NASA TN D-3782, 1967.

[18] D. Chubb, "Fully ionized quasi-one-dimensional magnetic nozzle flow," AIAA Journal, vol. 10, no. 2, pp. 113-114, 1972.

[19] M. A. Raadu, "Expansion of a plasma injected from an electrodeless gun along a magnetic field," Plasma Physics, vol. 21, no. 4, p. 331, 1979.

[20] R. Gerwin, G. Marklin, A. Sgro, and A. Glasser, "Characterization of plasma flow through magnetic nozzles," Tech. Rep. AFSOR AL-TR-89092, Los Alamos National Laboratory, 1990. 
[21] J. Sercel, "Simple model of plasma acceleration in a magnetic nozzle," in 21st International Electric Propulsion Conference, vol. 1, 1990.

[22] E. B. Hooper, "Plasma detachment from a magnetic nozzle," Journal of Propulsion and Power, vol. 9, no. 5, pp. 757-763, 1993.

[23] P. Mikellides, P. Turchi, and N. Roderick, "Applied-field magnetoplasmadynamic thrusters, part 1: Numerical simulations using the MACH2 code," Journal of Propulsion and Power, vol. 16, no. 5, 2000.

[24] E. Ahedo and M. Merino, "Two-dimensional supersonic plasma acceleration in a magnetic nozzle," Physics of Plasmas, vol. 17, p. 073501, 2010.

[25] E. Ahedo and M. Merino, "On plasma detachment in propulsive magnetic nozzles," Physics of Plasmas, vol. 18, p. 053504, 2011.

[26] W. Cox, C. Charles, R. Boswell, and R. Hawkins, "Spatial retarding field energy analyzer measurements downstream of a helicon double layer plasma," Applied Physics Letters, vol. 93, no. 7, p. 071505, 2008.

[27] C. Deline, R. Bengtson, B. Breizman, M. Tushentsov, J. Jones, D. Chavers, C. Dobson, and B. Schuettpelz, "Plume detachment from a magnetic nozzle," Physics of Plasmas, vol. 16, no. 3, p. 033502, 2009.

[28] K. Terasaka, S. Yoshimura, K. Ogiwara, M. Aramaki, and M. Tanaka, "Experimental studies on ion acceleration and stream line detachment in a diverging magnetic field," Physics of plasmas, vol. 17, no. 7, p. 072106, 2010.

[29] J. P. Squire, C. S. Olsen, F. R. Chang-Díaz, L. D. Cassady, B. W. Longmier, M. G. Ballenger, M. D. Carter, T. W. Glover, and G. E. McCaskill, "VASIMR VX-200 operation at $200 \mathrm{~kW}$ and plume measurements: Future plans and an ISS EP test platform," in 32nd International Electric Propulsion Conference, 2011.

[30] K. Takahashi, Y. Itoh, and T. Fujiwara, "Operation of a permanentmagnets-expanding plasma source connected to a large-volume diffusion chamber," Journal of Physics D: Applied Physics, vol. 44, no. 1, p. 015204, 2011.

[31] C. Olsen, M. Ballenger, M. Carter, F. Chang Diaz, M. Giambusso, T. Glover, A. Ilin, J. Squire, B. Longmier, E. Bering, and P. Cloutier, "Investigation of plasma detachment from a magnetic nozzle in the plume of the vx-200 magnetoplasma thruster," Plasma Science, IEEE Transactions on, vol. 43, no. 1, pp. 252-268, 2015.

[32] R. Moses, R. Gerwin, and K. Schoenberg, "Resistive plasma detachment in nozzle based coaxial thrusters," in Proceedings Ninth Symposium on Space Nuclear Power Systems, Albuquerque, New Mexico, 1992, AIP Conference Proceedings No. 246, pp. 1293-1303, 1992. 
[33] A. Arefiev and B. Breizman, "Magnetohydrodynamic scenario of plasma detachment in a magnetic nozzle," Physics of Plasmas, vol. 12, no. 5, p. 043504, 2005.

[34] E. Ahedo and M. Merino, "Two-dimensional plasma expansion in a magnetic nozzle: separation due to electron inertia," Physics of Plasmas, vol. 19, p. 083501, 2012.

[35] M. Merino and E. Ahedo, "Plasma detachment in a propulsive magnetic nozzle via ion demagnetization," Plasma Sources Science and Technology, vol. 23, p. 032001, 2014.

[36] J. M. Little and E. Y. Choueiri, "Thrust and efficiency model for electrondriven magnetic nozzles," Physics of Plasmas, vol. 20, no. 10, p. 103501, 2013.

[37] E. Ahedo and J. Navarro, "Helicon thruster plasma modeling: Twodimensional fluid-dynamics and propulsive performances," Physics of Plasmas, vol. 20, no. 4, p. 043512, 2013.

[38] L. Tonks, "Theory of magnetic effects in the plasma of an arc," Physical Review, vol. 56, no. 4, pp. 360-373, 1939.

[39] S. Chandrasekhar, "Axisymmetric magnetic fields and fluid motions," The Astrophysical Journal, vol. 124, no. 7, p. 232, 1956.

[40] M. J. Zucrow and J. D. Hoffman, Gas Dynamics (volume I), vol. I. John Wiley \& sons, 1976.

[41] M. Merino, F. Cichocki, and E. Ahedo, "Collisionless plasma thruster plume expansion model," Plasma Sources Science and Technology, vol. 24, no. 3, p. 035006, 2015.

[42] K. Takahashi, T. Lafleur, C. Charles, P. Alexander, and R. Boswell, "Electron diamagnetic effect on axial force in an expanding plasma: Experiments and theory," Physical Review Letters, vol. 107, no. 23, p. 235001, 2011.

[43] M. Merino and E. Ahedo, "Simulation of plasma flows in divergent magnetic nozzles," IEEE Transactions on Plasma Science, vol. 39, no. 11, pp. 29382939, 2011.

[44] M. Merino and E. Ahedo, "Fully magnetized plasma flow in a magnetic nozzle," Plasma Sources Science and Technology, (submitted for publication).

[45] G. Sutton and O. Biblarz, Rocket propulsion elements. Wiley, 2010.

[46] I. Boyd and R. Dressler, "Far field modeling of the plasma plume of a Hall thruster," Journal of applied physics, vol. 92, no. 4, p. 1764, 2002. 
[47] I. Boyd and A. Ketsdever, "Interactions between spacecraft and thruster plumes," Journal of Spacecraft and Rockets, vol. 38, no. 3, p. 380, 2001.

[48] J. Navarro and E. Ahedo, "Hybrid simulation of a magnetic nozzle plume: exploring magnetized/demagnetized regimes," in $34^{\text {th }}$ International Electric Propulsion Conference, no. IEPC-2015-354, Electric Rocket Propulsion Society, 2015.

[49] M. Merino and E. Ahedo, "Influence of electron and ion thermodynamics on the magnetic nozzle plasma expansion," IEEE Transactions on Plasma Science, vol. 43, no. 1, pp. 244-251, 2015.

[50] J. M. Little and E. Y. Choueiri, "Influence of the applied magnetic field strength on flow collimation in magnetic nozzles," in $50^{\text {th }}$ AIAA/ASME/SAE/ASEE Joint Propulsion Conference 83 Exhibit, no. AIAA 2014-3912, (Washington DC), AIAA, 2014.

[51] J. Navarro, S. Correyero, and E. Ahedo, "Collisionless electron cooling on magnetized plasma expansions: advances on modelling," in $34^{\text {th }}$ International Electric Propulsion Conference, 2015.

[52] Y. Hu and J. Wang, "Electron properties in collisionless mesothermal plasma expansion: Fully kinetic simulations," Plasma Science, IEEE Transactions on, vol. 43, no. 9, pp. 2832-2838, 2015.

[53] F. Cichocki, M. Merino, E. Ahedo, Y. Hu, and J. Wang, "Fluid vs pic modeling of a plasma plume expansion," in $34^{\text {th }}$ International Electric Propulsion Conference, no. IEPC-2015-420, (Fairview Park, OH), Electric Rocket Propulsion Society, 2015.

[54] J. P. Sheehan, B. W. Longmier, E. A. Bering, C. S. Olsen, J. P. Squire, M. G. Ballenger, M. D. Carter, L. D. Cassady, F. R. C. Díaz, T. W. Glover, and A. V. Ilin, "Temperature gradients due to adiabatic plasma expansion in a magnetic nozzle," Plasma Sources Science and Technology, vol. 23, no. 4, p. 045014, 2014.

[55] R. H. Comfort, "The magnetic mirror force in plasma fluid models," in Modeling Magnetospheric Plasma, American Geophysical Union, 1988.

[56] J. Navarro, M. Martinez-Sanchez, and E. Ahedo, "Collisionless electron cooling in a magnetic nozzle," in 50th AIAA/ASME/SAE/ASEE Joint Propulsion Conference, (Cleveland, Ohio), AIAA, 2014.

[57] A. Arefiev and B. Breizman, "Ambipolar acceleration of ions in a magnetic nozzle," Physics of Plasmas, vol. 15, no. 4, p. 042109, 2008.

[58] A. Arefiev and B. Breizman, "Collisionless plasma expansion into vacuum: Two new twists on an old problem," Physics of Plasmas, vol. 16, no. 5, p. 055707, 2009. 
[59] P. Zhu and R. Boswell, "Observation of nonthermal electron tails in an RF excited argon magnetoplasma," Phys. Fluids B, vol. 3, no. 4, pp. 869-874, 1991.

[60] S. Cohen, X. Sun, N. Ferraro, E. Scime, M. Miah, S. Stange, N. Siefert, and R. Boivin, "On collisionless ion and electron populations in the magnetic nozzle experiment (MNX)," IEEE Transactions on Plasma Science, vol. 34, no. 3, pp. 792-803, 2006.

[61] C. Charles, "High density conics in a magnetically expanding helicon plasma," Applied Physics Letters, vol. 96, no. 5, pp. 051502-051502, 2010.

[62] E. Ahedo and M. Martínez-Sánchez, "Theory of a stationary current-free double layer in a collisionless plasma," Physical Review Letters, vol. 103, no. 13 , p. $135002,2009$.

[63] M. Merino and E. Ahedo, "Two-dimensional quasi-double-layers in twoelectron-temperature, current-free plasmas," Physics of Plasmas, vol. 20, p. $023502,2013$.

[64] G. Hairapetian and R. Stenzel, "Observation of a stationary, current-free double layer in a plasma," Physical review letters, vol. 65, no. 2, pp. 175178, 1990.

[65] C. Charles and R. Boswell, "Current-free double-layer formation in a highdensity helicon discharge," Applied Physics Letters, vol. 82, no. 9, p. 1356, 2003.

[66] N. Singh, "Current-free double layers: A review," Physics of Plasmas, vol. 18 , no. 12, p. 122105, 2011.

[67] M. Merino and E. Ahedo, "Plasma detachment mechanisms in a magnetic nozzle," in $47^{\text {th }}$ AIAA/ASME/SAE/ASEE Joint Propulsion Conference $\&$ Exhibit, no. AIAA-2011-5999, (Washington DC), AIAA, 2011.

[68] B. Roberson, R. Winglee, and J. Prager, "Enhanced diamagnetic perturbations and electric currents observed downstream of the high power helicon," Physics of Plasmas, vol. 18, no. 5, p. 053505, 2011.

[69] R. A. Gerwin, "Integrity of the plasma magnetic nozzle," tech. rep., NASA/TP—2009-213439, 2009.

[70] R. Winglee, T. Ziemba, L. Giersch, J. Prager, J. Carscadden, and B. Roberson, "Simulation and laboratory validation of magnetic nozzle effects for the high power helicon thruster," Physics of Plasmas, vol. 14, no. 6, p. 063501, 2007.

[71] R. Goldston and P. Rutherford, Introduction to Plasma Physics. Institute of Physics Publishing, Bristol, 1995. 
[72] E. Ahedo and M. Merino, "Preliminary assessment of detachment in a plasma thruster magnetic nozzle," in $46^{\text {th }}$ AIAA/ASME/SAE/ASEE Joint Propulsion Conference $\mathcal{E}$ Exhibit, no. AIAA 2010-6613, (Washington DC), AIAA, 2010.

[73] J. Ramos, "Fluid formalism for collisionless magnetized plasmas," Physics of Plasmas, vol. 12, no. 5, p. 052102, 2005.

[74] J. Ramos, "General expression of the gyroviscous force," Physics of plasmas, vol. 12, no. 11, p. 112301, 2005.

[75] J. Navarro, M. Merino, and E. Ahedo, "A fluiddynamic performance model of a helicon thruster," in $48^{\text {th }}$ AIAA/ASME/SAE/ASEE Joint Propulsion Conference $\mathcal{E}^{3}$ Exhibit, no. AIAA-2012-3955, (Washington DC), AIAA, 2012 .

[76] "Towards thrust vector control with a 3D steerable magnetic nozzle," in $34^{\text {th }}$ International Electric Propulsion Conference, no. IEPC-2015-414, (Fairview Park, OH), Electric Rocket Propulsion Society, 2015.

[77] R. H. Levy and F. W. French, "Plasma radiation shield: Concept and applications to space vehicles," Journal of Spacecraft and Rockets, vol. 5, no. 5 , pp. 570-577, 1968.

[78] C. Bombardelli, H. Urrutxua, M. Merino, E. Ahedo, and J. Peláez, "The ion beam shepherd: A new concept for asteroid deflection," $A A$, vol. 90, no. 1, pp. $98-102,2013$.

[79] M. Merino, E. Ahedo, C. Bombardelli, H. Urrutxua, and J. Peláez, "Ion beam shepherd satellite for space debris removal," in Progress in Propulsion Physics (L. T. DeLuca, C. Bonnal, O. J. Haidn, and S. M. Frolov, eds.), vol. IV of EUCASS Advances in Aerospace Sciences, ch. 8, pp. 789-802, Torus Press, 2013. 